Please do not remove this page

RMIT

UNIVERSITY

\title{
Computationally effective reasoning about goal interactions
}

Thangarajah, John; Padgham, Lin

https://researchrepository.rmit.edu.au/esploro/outputs/9921861709101341/filesAndLinks?institution=61RMIT_INST\&index=null

Thangarajah, J., \& Padgham, L. (2011). Computationally effective reasoning about goal interactions. Journal of Automated Reasoning, 47(1), 17-56. https://doi.org/10.1007/s10817-010-9175-0

Document Version: Accepted Manuscript

Published Version: https://doi.org/10.1007/s10817-010-9175-0

Repository homepage: https://researchrepository.rmit.edu.au

(c) Springer Science+Business Media B.V. 2010

Downloaded On 2023/04/26 18:00:18 +1000

Please do not remove this page 
Thank you for downloading this document from the RMIT Research Repository.

The RMIT Research Repository is an open access database showcasing the research outputs of RMIT University researchers.

RMIT Research Repository: http://researchbank.rmit.edu.au/

\section{Citation:}

Thangarajah, J and Padgham, L 2011, 'Computationally effective reasoning about goal interactions', Journal of Automated Reasoning, vol. 47, no. 1, pp. 17-56.

See this record in the RMIT Research Repository at:

https://researchbank.rmit.edu.au/view/rmit:6046

Version: Accepted Manuscript

Copyright Statement: (c) Springer Science+Business Media B.V. 2010

Link to Published Version:

http://dx.doi.org/10.1007/s10817-010-9175-0 


\title{
Computationally Effective Reasoning About Goal Interactions
}

\author{
John Thangarajah • Lin Padgham
}

Received: 5 June 2009 / Accepted: 12 April 2010 / Published online: 8 May 2010

(C) Springer Science+Business Media B.V. 2010

\begin{abstract}
It is important that intelligent agents are able to pursue multiple goals in parallel, in a rational manner. In this work we have described the careful empirical evaluation of the value of data structures and algorithms developed for reasoning about both positive and negative goal interactions. These mechanisms are incorporated into a commercial agent platform and then evaluated in comparison to the platform without these additions. We describe the data structures and algorithms developed, and the X-JACK system, which incorporates these into JACK, a state of the art agent development toolkit. There are three basic kinds of reasoning that are developed: reasoning about resource conflicts, reasoning to avoid negative interactions that can happen when steps of parallel goals are arbitrarily interleaved, and reasoning to take advantage of situations where a single step can help to achieve multiple goals. X-JACK is experimentally compared to JACK, under a range of situations designed to stress test the reasoning algorithms, as well as situations designed to be more similar to real applications. We found that the cost of the additional reasoning is small, even with large numbers of goal interactions to reason about. The benefit however is noticeable, and is statistically significant, even when the amount of goal interactions is relatively small.
\end{abstract}

Keywords Artificial intelligence - Goal interactions • Agent reasoning • Empirical evaluation $\cdot$ Conflict detection $\cdot$ Plan merging

J. Thangarajah $(\bowtie) \cdot$ L. Padgham

School of Computer Science, RMIT University, Melbourne, Australia

e-mail: john.thangarajah@rmit.edu.au

L. Padgham

e-mail: lin.padgham@rmit.edu.au 


\section{Introduction}

Agent oriented programming and agent development platforms that facilitate building intelligent agents are becoming increasingly popular. One common intelligent agent framework is the Belief Desire Intention model (BDI) [19]. Goals are a key concept in this model where a goal is a task that the agent wants to achieve. For example, a Personal Assistant agent may have goals to book a flight and schedule a meeting, or a Mars Rover agent may have goals to conduct experiments and recharge fuel.

This model however does not incorporate any reasoning about goal interactions. If a set of goals pursued in parallel contains inherent conflicts about resources, these are not noticed. Also it is possible for steps in achieving goals to be interleaved in such a way that necessary dependencies for goal achievement are violated.

The systems also do not take advantage of situations where a single step could assist in the achievement of more than one goal, if scheduled appropriately. We have developed data structures and algorithms that have been incorporated into the JACK system, in order to address these issues. JACK [5] is a BDI agent development platform.

This paper provides an overview of this additional reasoning, ${ }^{1}$ and its incorporation into JACK. It then provides a detailed and careful empirical analysis of the costs and benefits of the addition. We conclude that the computational costs are minimal, while the benefits are clearly significant_-both statistically and from the viewpoint of an application that needs to successfully achieve its goals. Our evaluation ascertains both the costs and the benefits of each of the additional reasoning mechanisms. The implementation contains all three mechanisms, and in practice they are all used together. However in the evaluation we chose to evaluate them separately as each requires a different analysis, and differing conditions for stress testing. We find that in all cases the cost of the additional reasoning is small, even with large numbers of goal interactions to reason about. The benefit however is noticeable and statistically significant, even when the number of interactions is relatively small. Adding this reasoning to an agent systems is therefore very worthwhile.

\subsection{Related Work}

Goals are an integral part of many agent development theories and frameworks $[13,14,18]$. However, until recently there has been fairly limited work on formalisms or representations for goals in agent systems that support reasoning algorithms that could be easily integrated into modern state of the art BDI agent implementation platforms such as JACK.

In terms of goal representation, the work of Hindriks et al. [11] has developed a new programming language called $\mathrm{GOAL}^{2}$ which captured the declarative aspect of goals, while more recent work of van Riemsdijk et al. [26] and our own previous

\footnotetext{
${ }^{1}$ Papers describing the specific data structures and algorithms for each of the reasoning modules have been published at the ECAI02 [25], AAMAS03 [24], and IJCAI03 [23] conferences.

${ }^{2}$ Goal Oriented Agent Language.
} 
work $^{3}$ [27] captured both the declarative as well as procedural aspects of goals. Bell and Huang [2] introduced and formalised goal hierarchies. This literature however did not present detailed reasoning algorithms about goal interactions.

In the multi-agent setting the work of Barber et al. [1] on conflict representation and of Clement and Durfee [6-8] on co-ordinating concurrent hierarchical planning agents using summary information, reasoned about negative goal interactions. The work of Cox and Durfee [9] on discovering and exploiting synergy between hierarchical planning agents address reasoning about positive goal interactions.

There is also a body of work on combining the concepts of distributed transactions to multi-agent systems to provide reliable computational models $[4,17]$. They have provided extensions to the BDI model to allow for transaction based reasoning [4]. Whilst this work is useful to ensure that steps, in particular concurrent steps, taken by an agent or a team of agents are consistent, it does not deal with synergies or potential conflicts. Our approach does not always guarantee a reliable system but rather we provide mechanisms by which conflicts and synergies (both definite and potential) may be identified using the standard BDI model.

In the single-agent setting work by Horty and Pollack [12] on evaluating new options in the context of existing plans addresses the issue of positive interactions between plans. Although this is formalised for plans, it is conceptually useful in the context of goals as plans are used to achieve goals.

We have developed data structures and algorithms that support three specific kinds of reasoning about goals: management of goals that conflict due to resource constraints [25], management of situations where a state needs to be protected until some steps towards a goal have happened [23], and reasoning to take advantage of situations where achieving some steps in different goals can be combined [24]. In contrast to related work in a multi-agent setting, where conflict detection is inherently complex due to the fact that agents do not necessarily share the knowledge of other agents, the fact that we are working on reasoning within a single agent enables our algorithms to access the internal data of the agent allowing them to be simpler and more efficient.

There are some similarities in the data structures and intuitions underlying the reasoning, between our work and that of Clement and Durfee [6-8] on co-ordinating hierarchical plans in societies of agents. They have also done experimental analysis of the efficiency of their algorithms [8]. However, they do not analyse benefits in terms of goal completions as we do, but rather in terms of solution cost. Also their approach is somewhat different to ours in that they are developing a plan incorporating all goals (or tasks), while we are simply enabling an agent to avoid making foolish decisions about which goals it can/should pursue. Our aims are in some ways more limited, hence we are able to realise greater efficiency.

In Section 2 we review the data structures which underlie each of the algorithms. In Section 3 we then briefly describe the three different kinds of reasoning. More detail on this aspect can be found in the relevant conference papers [23-25]. The specific toolkit we have chosen is JACK which is a Java based state of the art agent development toolkit that has been used to develop a variety of large scale

\footnotetext{
${ }^{3}$ This work was done in collaboration with Michael Winikoff and James Harland, who are also members of the Intelligent Agents group at RMIT.
} 
applications. In Section 4 we describe our extension to JACK (which we will call $\mathrm{X}$-JACK-extended JACK) to incorporate an explicit structure for goals and the associated reasoning. In Section 5 we then compare JACK and X-JACK in a range of situations, on each of the algorithms, measuring both the computational efficiency, by the time taken, and the effectiveness in terms of number of goals successfully completed. In addition to indicating that this additional reasoning is not computationally expensive, our evaluation and analysis also provides an indication of the kind of domains for which goal-resource related reasoning is most important.

\section{Representing Goals and Plans}

In this section we present the representation of goals and plans that allow the agent to reason about goal interactions. The structure for plans is important because plans play a key role in the reasoning process, since goals are achieved via plans. An important feature of these structures is their ability to be easily implemented in practical agent systems. Many of these data structures are common to a broad range of BDI systems. However, we build on these, both in making explicit the existing implicit tree structure of goals and plans, so that it can be traversed by our algorithms, and by adding specific information, some of which is calculated automatically.

In the same manner in which a class is defined as a template for an object in an object-oriented programming language [3,20], a goal-type is introduced as a template for a goal. When an agent chooses to pursue a particular goal, a goal-instance of the goal-type is created (similar to how an object is instantiated from a class, in objectoriented terms). Similarly, a plan-type is defined as a template for a plan, and a planinstance is created when the agent executes a particular plan-type.

Below is the Bachus-Naur Form (BNF) of the goal-type (GoalType) and plantype (PlanType) structures as used in this work, followed by a discussion of their attributes. $^{4}$

$\langle$ GoalType $\rangle \rightarrow\langle$ GoalTypeName $\rangle\{\langle$ Plans $\rangle\}\{\langle$ In-Conditions $\rangle\}\{\langle$ Effects $\rangle\}$

$\langle$ GoalTypeName $\rangle \rightarrow\langle$ Label $\rangle$

$\langle$ Plans $\rangle \rightarrow\langle$ PlanTypeName $\rangle(,\langle\text { PlanTypeName }\rangle)^{*}$

$\langle$ PlanTypeName $\rangle \rightarrow\langle$ Label $\rangle$

$\langle$ In-Conditions $\rangle \rightarrow \lambda \mid\langle$ Condition $\rangle(\text {, }\langle\text { Condition }\rangle)^{*}$

$\langle$ Effects $\rangle \rightarrow \lambda \mid\langle$ Condition $\rangle(,\langle\text { Condition }\rangle)^{*}$

$\langle$ Condition $\rangle \rightarrow\langle$ Statement $\rangle \mid$ NOT $\langle$ Statement $\rangle$

$\langle$ Statement $\rangle \rightarrow$ string $\mid\langle$ Variable $\rangle=\langle$ Value $\rangle$

$\langle$ PlanType $\rangle \rightarrow\langle$ PlanTypeName $\rangle\{\langle$ Pre-Conditions $\rangle\}\{\langle$ In-Conditions $\rangle\}\{\langle$ Effects $\rangle\}$ $\{\langle$ PlanBody $\rangle\}\{\langle$ Resources $\rangle\}$

$\langle$ Pre-Conditions $\rangle \rightarrow \lambda \mid\langle$ Condition $\rangle(\text {, }\langle\text { Condition }\rangle)^{*}$

$\langle$ PlanBody $\rangle \rightarrow\langle$ Action $\rangle \mid\langle$ GoalTypeName $\rangle \mid(\langle$ PlanBody $\rangle$; $\langle$ PlanBody $\rangle) \mid$ $(\langle$ PlanBody $\rangle \|\langle$ PlanBody $\rangle)$

$\langle$ Resources $\rangle \rightarrow \lambda \mid\langle$ Resource $\rangle(,\langle\text { Resource }\rangle)^{*}$

${ }^{4}$ Note that the goal, plan and condition representations should be viewed as sentence schemas, that is, they may have arguments, but there is no quantification over variables. 


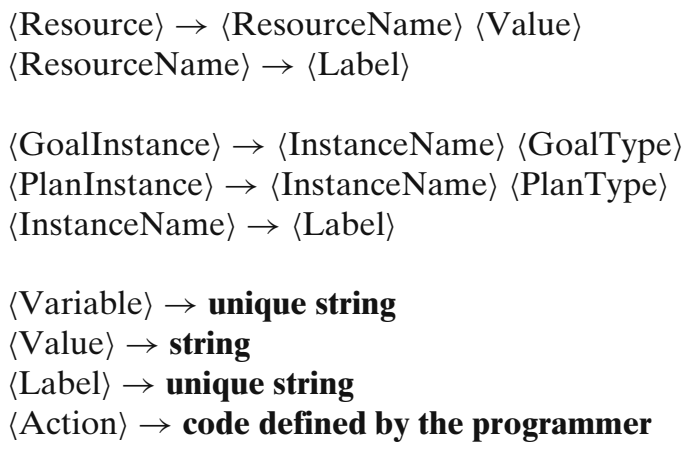

- GoalTypeName and PlanTypeName-A label which indicates the type of the goal or plan respectively. e.g. GoToBeachGoal, GoToBeachPlan.

- Plans-The set of plan-types that could possibly satisfy the goal. An agent could have more than one plan that achieves the same goal, but for every goal there must be at least one plan that could satisfy the goal. Therefore, this set of plantypes cannot be empty. Whilst having a plan does not necessarily imply that the goal pursuit will be successful, it states that the goal is possible under certain conditions as determined by the appropriate plan. e.g. GoToBeachGoal.Plans(TaxiToBeachPlan, WalkToBeachPlan, ..).

- In-Conditions - The set of conditions that must hold during the pursuit of a goal/plan. If any of the in-conditions become false during the pursuit/execution of the goal/plan, the goal/plan is dropped with failure.

e.g. GoToBeachGoal.InConditions(WeatherIsSunny, not FeelingLazy). TaxiToBeachPlan.inConditions(HaveMoney).

- Effects-For a goal, this is the set of conditions that must be achieved in order for the goal to succeed. e.g. GoToBeachGoal.Effects(AtBeach).

For a plan, the effects are the set of conditions that are made true by the actions within the plan. This set does not include the effects of any sub-goals. This is because the programmer cannot pre-determine the run-time path that might be chosen to satisfy the sub-goals, which determines their effects. Attaching effects to plans rather than actions allows the programmer to write arbitrary code within the plan body, which is similar to that done in practical systems like JACK. The onus is on the designer of the agent system to ensure that all relevant effects are identified for goals and plans. However, this is relatively straightforward as for a plan the designer only needs to include the effects of its actions and not the effects of its sub-goals. Similarly for a goal, only the desired end effects of the goal need to be included.

e.g. TaxiToBeachPlan.effects (AtBeach, OweTaxiDriver $(\$ 20)$ ).

The effects declared at each goal are the intended effects of that goal (also termed as primary expectations by Pollack [16]), ${ }^{5}$ and the effects of a plan that

\footnotetext{
${ }^{5}$ Pollack defines expectations as beliefs about future activities and circumstances. Primary expectations are those that are directly intended by the agents actions and secondary expectations are those that are side effects of intended actions.
} 
are not effects of the corresponding goal, are side effects of the goal (secondary expectations). For example, the plan to take a taxi to the beach may achieve the effects AtBeach and OweTaxiDriver (\$20). By examining the effects of the goal (i.e. AtBeach) it can be determined that OweTaxiDriver $(\$ 20)$ is a side-effect as it is not an intended effect of the goal.

- Pre-Conditions-Are specific to plans and are the set of conditions that must be true in order for an instance of the plan to begin execution. The conditions need not hold once the plan has begun execution.

e.g. TaxiToBeachPlan.preCondition(AtTaxiStand, HaveMoney).

The pre-conditions are implicitly extended with the in-conditions. For example, the in-condition of HaveMoney is also included in the pre-conditions. If it is not included and AtTaxiStand is true but HaveMoney is false, the plan would begin, as the pre-condition is true, but fail immediately since the in-condition is not true.

- PlanBody-This represents the functionality of the plan and contains actions and sub-goals. These actions and sub-goals are combined by either sequencing them (e.g. "achieve goal $G_{1}$ and then perform action $A$ ", written as " $G_{1} ; A$ ") or by performing them in parallel (e.g. "achieve goals $G_{1}$ and $G_{2}$ ", written as " $G_{1} \| G_{2}$ "). All actions are assumed to be atomic, while sub-goals are treated the same as top-level goals.

e.g. $\operatorname{body}($ TaxiToBeachPlan) $::=$

\section{HailTaxiAction; Negotiate FareGoal; PayFareGoal}

- Resources-Are specific to plans, as they determine the resources required to achieve a goal. This attribute captures the resource requirements of all actions within the plan and does not include the resource requirements of any sub-goals of the plan.

e.g. TaxiToBeachPlan.Resources(\{(Money, 100)\}).

- InstanceName - This is an additional attribute that is attached to each goal and plan instance. The instance-name is a label that provides a unique handle to each goal/plan instance, as there can be multiple instances of the same goal/plan type. While all goal/plan type attributes are pre-assigned to each goal/plan type at compile-time, the instance name is assigned at run-time.

Within the general BDI framework, goals are achieved by executing plans and each goal has at least one plan, if not many, that can be used to satisfy the goal. Each plan can include sub-goals, but need not have any. This relationship between a top level goal, its plans and sub-goals defines a tree structure for each top-level goal, which is termed the goal-plan tree for that goal.

In this goal-plan tree, goals are represented as goal-nodes (GoalNode) and plans as plan-nodes (PlanNode). Each node contains a reference to the goal-type or plantype that it represents. Therefore, the attributes of the relevant goal or plan can be accessed at each node.

The root of a goal-plan tree is a top-level goal, and its children are its potential plans. For each plan-node, its children are the sub-goals of the plan. Recall that the sub-goals may be combined in sequence or in parallel with each other. The sequenceoperator (;) and parallel-operator $(\|)$ are used to denote this in the tree. Figure 1 is an example goal-plan tree for a goal to go to the beach (GoToBeachGoal).

The agent only needs to execute one of the plans in order to satisfy the goal. Therefore, in the tree structure the children of a goal-node (i.e. the plan-nodes) are viewed as "OR" nodes. On the other hand, for a particular plan to succeed, all the 


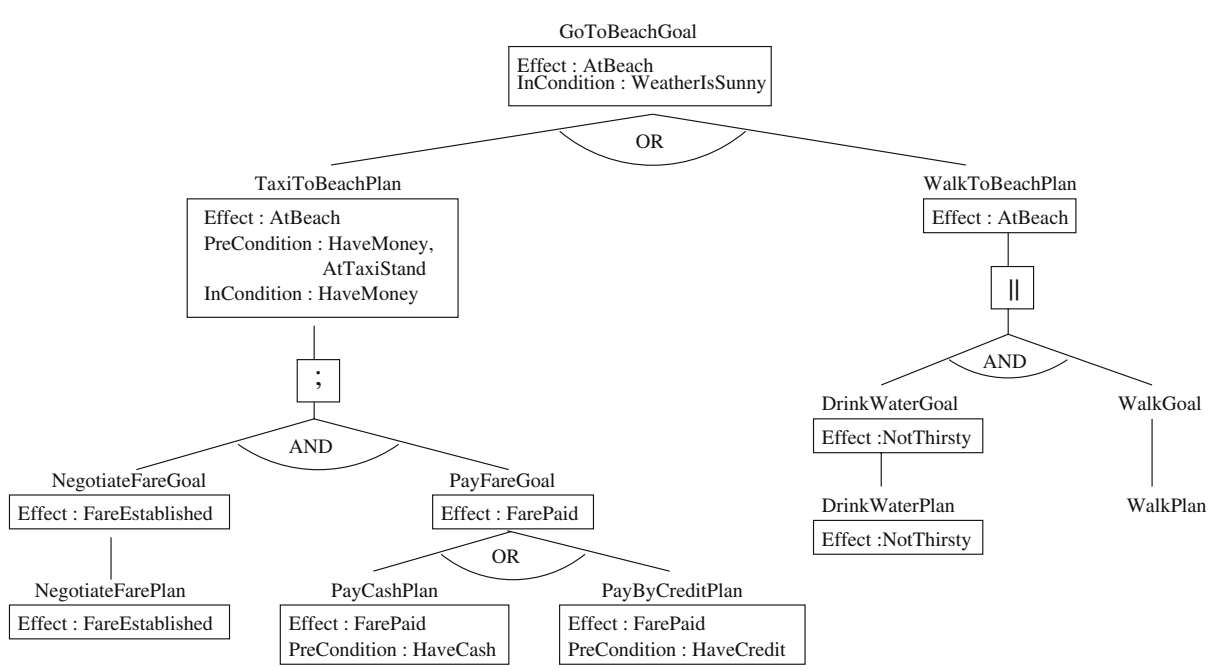

Fig. 1 An example of a goal-plan tree

sub-goals of a plan (if any), irrespective of whether they are in sequence or in parallel, must be attempted and succeed. Hence, the children of a plan-node (i.e. the goalnodes) are viewed as "AND" nodes, in the tree. The leaf nodes of the tree are plannodes with no children. In other words, they are plans that have no sub-goals.

Actions are not represented within the tree structure, because they are considered to be atomic and their properties such as the pre-conditions, in-conditions and effects are abstracted to the plan level.

When the agent system is compiled, the goal-plan tree templates for each goal are created and when the agent decides to pursue a goal, a goal-plan tree instance is created at run-time. As an agent executes its plans and sub-goals this tree is updated dynamically to reflect its progress. For example, if we assume the agent with the goal of GoToBeachGoal chose the TaxiToBeachPlan and has completed NegotiateFarePlan and begun the PayByCreditPlan, the tree would be annotated as shown in Fig. 2. This allows the agent to monitor the progress of its goals.

One of the key features that this tree structure provides is that it allows information about (sub)goals and plans at the lower levels of the hierarchy to be propagated to higher levels all the way up to the root top-level goal-node. This propagated information stored at each node-level is termed summary information.

The summary information can be used to detect interactions between goals. For example, in the goal-plan tree for GoToBeachGoal (Fig. 1), it is possible to propagate the fact that TaxiToBeachPlan requires the pre-condition of HaveMoney to the top-level goal-node. The agent would then know that there is a possibility that it may require HaveMoney in order to satisfy GoToBeachGoal. It is only a possibility because it depends on the choice of plan. If HaveMoney was required no matter which way the agent chose to achieve GoToBeachGoal, then we say HaveMoney is definitely required.

If the agent then decides to pursue another goal that causes it to lose all its money (i.e. not HaveMoney), it can use the summary information to realise that there is a 


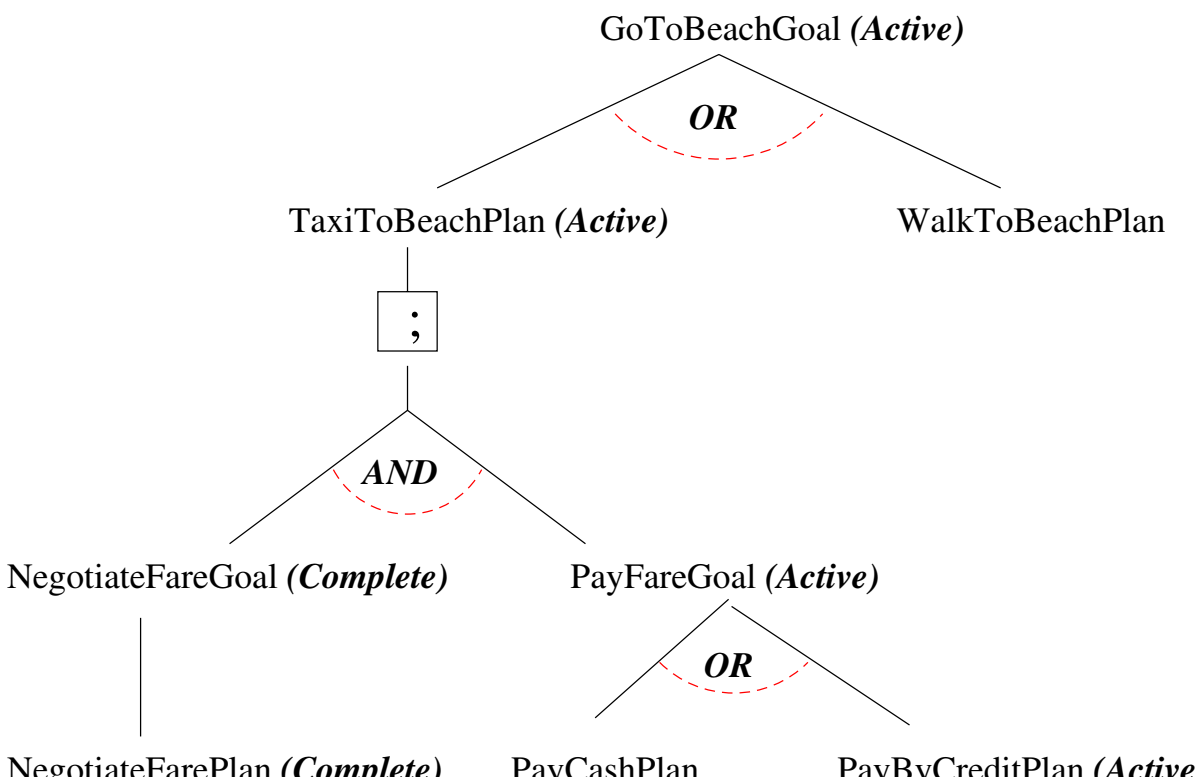

NegotiateFarePlan (Complete) PayCashPlan PayByCreditPlan (Active)

Fig. 2 Dynamically updating a goal-plan tree

possible conflict. This does not require the agent to examine all the plans of each goal, the sub-goals of each plan and so on, which can be a very costly operation. This is the fundamental concept that is used in our algorithms that discover interactions between goals.

Apart from pre-condition, in-condition and effect summaries at each node, additional information is summarised according to the requirements of the reasoning methods.

\section{Reasoning Mechanisms}

We have developed three different aspects of reasoning about goal interactions. The first detects situations where conflicts regarding resources required by parallel executing goals could cause failure of one or both goals. The second deals with situations where careless interleaving of steps to achieve goals may cause a goal to fail. The third is concerned with situations where it may be possible to make use of a single step to achieve more than one goal, if it is scheduled appropriately.

In order to provide the reader with an insight into the nature and complexity of the algorithms that we have developed, we present some of the details on the mechanisms for resource conflict management [25] in Section 3.1 below. For the other two aspects however, we present an overview of the reasoning rather than the details as the approaches are similar to that of resource conflict management in terms of the techniques used. The details of these mechanisms can be found in [24] and [23], respectively. 


\subsection{Resource Conflict Management}

Agents created in real world applications may use various kinds of resources to satisfy their goals. For example, a Mars rover agent with the goal of performing experiments on soil samples on the surface of Mars may require resources such as energy (fuel) to power on-board machinery, and a communication channel to transmit experimental results.

Agents typically pursue multiple goals, some being pursued concurrently. The resources available to these agents are usually finite. When multiple goals compete for these finite resources, conflicting situations are not uncommon. For example, two goals that require 100 units of energy each cannot be successfully pursued together, if only 150 units of energy are available. There is a risk that if both goals are attempted neither may succeed. A rational agent should identify such conflicts and at least not attempt to pursue goals that will inevitably conflict.

In order to reason about potential resource conflicts, plans and goals in the goalplan tree are annotated with summarised information about needed resources, which are classified as consumable or re-usable. Re-usable resources are made available after they have been used but consumable resources are not. For example, fuel is a consumable resource, and a communication channel is a reusable resource.

Actual resource usage will depend on which plans the agent attempts to use to achieve its goals. Consequently resource summaries specify necessary resources ${ }^{6}$ of that goal, which are required no matter which way the goal is achieved, and possible resources, which may be required, depending on actual plan choices.

The creation of the goal-plan tree and the derivation of resource summaries for plans and goals is done at compile time. We provide the details of how these summaries are derived below:

\subsubsection{Deriving Resource Summary Information}

Resource requirements for a goal are derived by combining the resource requirements of all relevant plans for that goal. The resource requirements of a plan are calculated by combining resource requirements of the sub-goals and actions within the plan body.

Resource requirements are not attached to each individual action in the system, but a summary of the resource requirements of all the actions within a plan is specified by the programmer at each plan. ${ }^{7}$ These shall be referred to as the resource requirement of the plan, and denoted by the symbol $P^{\mathcal{R}}$ for a plan $P$. Note that $P^{\mathcal{R}}$ does not contain the resource requirements of the sub-goals within the plan, but only of its actions. For example, the resource requirements of the Mars rover agent's plan to transmit the results to the lander (TransmitTo(Lander)Plan) are $\{($ Energy, 50) $\}$ i.e. TransmitTo $($ Lander $)$ Plan $^{\mathcal{R}}=\{($ Energy, 50) $\}$.

\footnotetext{
${ }^{6}$ Note that having all necessary resources does not mean that sufficient resources are available to achieve the goal. For example, if plan $\mathrm{A}$ for achieving $\mathrm{G}$ requires resources $\mathrm{X}$ and $\mathrm{Y}$, and plan $\mathrm{B}$ requires resources $\mathrm{Y}$ and $\mathrm{Z}$, then we can conclude that $\mathrm{Y}$ is a necessary resource. However sufficient resources for successful achievement of $\mathrm{G}$ would be ( $\mathrm{Y}$ and $(\mathrm{X}$ or $\mathrm{Z})$ ).

${ }^{7}$ The reason for this is that it gives a better mapping to implementations, where a plan may consist of arbitrary code plus sub-goals, rather than the simpler formalisation given here. It also relieves the programmer from the need to specify resource requirements at the level of each action.
} 
In order to facilitate the computation of resource summaries of goals and plans, a number of operators over resource sets need to be defined.

$\sqcup$ and $\sqcap$ operators The $\sqcup$ operator computes the upper bound (maximum) of two resource sets. For example, if

$$
\begin{gathered}
R_{1}=\{(\text { Energy }, 50),(\text { ComCh }, 1)\} \text { and } R_{2}=\{(\text { Energy }, 100)\} \text { then } \\
R_{1} \sqcup R_{2}=\{(\text { Energy }, 100),(\text { ComCh }, 1)\} .
\end{gathered}
$$

The $\sqcap$ operator computes the lower bound (minimum). Using the same example, $R_{1} \sqcap R_{2}=\left\{(\text { Energy, 50) }\}^{8}\right.$

Formally:

$$
\begin{aligned}
& R_{1} \sqcup R_{2}=\left\{\left(t, \max \left(\#\left(R_{1}, t\right), \#\left(R_{2}, t\right)\right)\right) \mid t \in \mathcal{T}\right\} \\
& R_{1} \sqcap R_{2}=\left\{\left(t, \min \left(\#\left(R_{1}, t\right), \#\left(R_{2}, t\right)\right)\right) \mid t \in \mathcal{T}\right\}
\end{aligned}
$$

$\oplus$ and $\otimes$ operators $\quad$ When determining the resource needs of a plan, there is a need to distinguish between the sub-goals within a plan that are achieved in sequence and those that are achieved in parallel. If two goals $G_{1}$ and $G_{2}$ are done in parallel (i.e. $G_{1} \| G_{2}$ ), then the resources of each goal can be combined by a simple addition of the resource sets, irrespective of whether the resources are consumable or reusable. This operation is denoted by the $\oplus$ operator. For example, if the necessary resource requirements of $G_{1}$ and $G_{2}$ are $N_{1}$ and $N_{2}$ respectively and

$$
\begin{gathered}
N_{1}=\{(\text { Energy }, 50),(\text { ComCh }, 1)\} \text { and } N_{2}=\{(\text { Energy }, 100),(\text { ComCh }, 1)\} \text { then } \\
N_{1} \oplus N_{2}=\{(\text { Energy }, 150),(\text { ComCh }, 2)\}
\end{gathered}
$$

If $G_{1}$ and $G_{2}$ are done in sequence (i.e. $G_{1} ; G_{2}$ ), then addition would provide the correct combined requirement for resources that are consumable. However, because re-usable resources are available after use, the combined requirement of two sequential sub-goals is calculated using the upper bound $(\sqcup)$ operator. This combination operation is denoted by the $\otimes$ operator.

$$
N_{1} \otimes N_{2}=\{(\text { Energy }, 150),(\text { ComCh }, 1)\}
$$

Formally, on any resource sets $R_{1}$ and $R_{2}$ :

$$
\begin{aligned}
& R_{1} \oplus R_{2}=\left\{\left(t, \#\left(R_{1}, t\right)+\#\left(R_{2}, t\right)\right) \mid t \in \mathcal{T}\right\} \\
& R_{1} \otimes R_{2}=\left(R_{1}^{c} \oplus R_{2}^{c}\right) \cup\left(R_{1}^{r} \sqcup R_{2}^{r}\right)
\end{aligned}
$$

The possible resources of sub-goals are also determined in a similar manner. In general the resource summaries of two sub-goals that are executed in parallel are combined as:

$$
\mathcal{S}\left(G_{1} \| G_{2}\right)=\mathcal{S}\left(G_{1}\right) \oplus \mathcal{S}\left(G_{2}\right)=\left\langle N_{1}, P_{1}\right\rangle \oplus\left\langle N_{2}, P_{2}\right\rangle=\left\langle N_{1} \oplus N_{2}, P_{1} \oplus P_{2}\right\rangle
$$

\footnotetext{
${ }^{8}$ Note that the result is the normalised form of $\{($ Energy, 50$),($ Com Ch, 0$)\}$.
} 
and if they are executed in sequence as follows:

$$
\mathcal{S}\left(G_{1} ; G_{2}\right)=\mathcal{S}\left(G_{1}\right) \otimes \mathcal{S}\left(G_{2}\right)=\left\langle N_{1}, P_{1}\right\rangle \otimes\left\langle N_{2}, P_{2}\right\rangle=\left\langle N_{1} \otimes N_{2}, P_{1} \otimes P_{2}\right\rangle .
$$

$\uplus$ operator In computing the possible (maximum) resource requirements of a goal it is important to note that it may be necessary for more than one plan to be executed in order to achieve that goal, due to plan failure. Note also that consumable resources that have been used in this process cannot be recovered. Consequently, the possible resource needs of the goal must combine the resource needs of all relevant plans, as if each were to be executed sequentially applying the $\otimes$ operator as shown above.

The necessary resources of a goal are the minimum amount for each resource across all relevant plans of the goal and can be derived using the lower bound $(\sqcap)$ operator.

The $\uplus$ operator can be defined to combine the resource requirements of plans as follows:

$$
\left\langle N_{1}, P_{1}\right\rangle \uplus\left\langle N_{2}, P_{2}\right\rangle=\left\langle N_{1} \sqcap N_{2}, P_{1} \otimes P_{2}\right\rangle
$$

For example, if two plans $P L_{1}$ and $P L_{2}$ have the resource summaries of $S\left(P L_{1}\right)$ and $S\left(P L_{2}\right)$ respectively, and

$$
\begin{gathered}
S\left(P L_{1}\right)=\langle\{(\text { Energy }, 30)\},\{(\text { Energy }, 50),(\text { Com Ch }, 1)\}\rangle \text { and } \\
S\left(P L_{2}\right)=\langle\{(\text { Energy }, 20)\},\{(\text { Energy }, 20),(\text { ComCh }, 1)\}\rangle \text { then } \\
S\left(P L_{1}\right) \uplus S\left(P L_{2}\right)=\langle\{(\text { Energy }, 20)\},\{(\text { Energy }, 70),(\text { Com } C h, 1)\}\rangle .
\end{gathered}
$$

In the above case, the necessary energy is 20 units because that is the minimum energy required to achieve the goal (i.e. by choosing the plan $P L_{2}$ ). The possible energy required is 70 units because if the plan $P L_{2}$ is chosen and fails, and is then followed by the execution of the plan $P L_{1}$, the total possible units of energy required is 70 units. Since the resource $\mathrm{ComCh}$ is a re-usable resource, even though it is possibly required by both $P L_{1}$ and $P L_{2}$, it can only be required by one at a time, as the plans are alternatives.

Having defined all of the above operators, we now define how the resource summaries of a goal can be computed, by combining the resource summaries of its components. The lifting of the operators $\oplus, \otimes, \sqcap$, and $\sqcup$ is defined to operate on pairs of resources in the obvious way. For example,

$$
\left\langle N_{1}, P_{1}\right\rangle \oplus\left\langle N_{2}, P_{2}\right\rangle=\left\langle N_{1} \oplus N_{2}, P_{1} \oplus P_{2}\right\rangle .
$$

The resource requirements of a goal $G$ and a plan $P L$ are denoted by $\mathcal{S}(G)$ and $\mathcal{S}(P L)$ respectively. Note that they are each a two tuple $\langle N, P\rangle$. The set of relevant plans for a goal $G$ is denoted by plans-of $(G) . P L^{\mathcal{R}}$ contains the resource requirements of all the actions within the plan body of $P L$ and hence the resource requirements of individual actions are not considered in the function below. Also, recall from our representation for plans (see Section 2) that sub-goals within a plan 
body can be either in sequence (;), in parallel $(\|)$, or in a nested combination of the two.

$$
\begin{aligned}
\mathcal{S}(G) & =\biguplus_{p \in \text { plans }-o f(G)} \mathcal{S}(p) \\
\mathcal{S}(P L) & \left.=\left\langle P L^{\mathcal{R}}, P L^{\mathcal{R}}\right\rangle \oplus \mathcal{S}(\text { PL.planBody })\right) \\
\mathcal{S}\left(G_{1} \| G_{2}\right) & =\mathcal{S}\left(G_{1}\right) \oplus \mathcal{S}\left(G_{2}\right) \\
\mathcal{S}\left(G_{1} ; G_{2}\right) & \left.=\mathcal{S}\left(G_{1}\right) \otimes \mathcal{S}\left(G_{2}\right)\right)
\end{aligned}
$$

The algorithm for generating the goal-plan tree with annotated resource summaries is shown in Fig. 3. In the figure, the procedure generate Tree creates a goal-plan tree for a particular goal and also derives and attaches the resource summary at each node.

$\mathcal{S}^{\prime}$ and $\mathcal{S}^{\prime \prime}$ only considers the resource summaries of the child nodes. This is efficient, since only the children of the node (that is the argument to these functions) are considered, only a single computation needs to be performed; namely combining the child nodes' summaries with the appropriate function $(\oplus, \otimes$, or $\uplus$, depending on the nature of node).

\subsubsection{Dynamic Updates of Resource Requirements}

Given that the possible resource requirements of a goal considers the worst case situation, initially ${ }^{9}$ there is likely to be a wide spread of values between the necessary and possible resource requirements. This spread however, narrows dynamically as plans get executed and either succeed or fail. In this section we will describe a mechanism that allows the resource summaries to be dynamically updated at runtime.

In pursuing a goal $G$, once a plan $P$ of $G$ has completed, all possible and necessary resources associated with that plan are no longer relevant. Also with respect to the resource tree of $G$, when $P$ completes, the summary information of the nodes (i.e. goal and plan nodes) above $P$ in the tree will no longer be accurate. For example, in Fig. 5, if plan Collect(Soil)Plan completes, the resource summaries of the nodes above it will change as shown in Fig. 5 below.

Reasoning about the remaining resource requirements of partially achieved goals can enable an agent to make more up-to-date choices regarding its adoption of goals. For example, take the goal $G_{1}$ from Fig. 5, and a new goal the agent is considering, $G_{2}$, with resource summary $\langle\{\emptyset\},\{($ Energy, 75) $\}\rangle$. If the current resource availability (i.e. after Collect(Soil)Plan of $G_{1}$ completes) is $\mathcal{R}=\{($ Energy, 470) $\}$ then using the updated resource summary of $G_{1}$ the agent can deduce that it is safe to execute $G_{2}$ in parallel with $G_{1}\left(\oplus P_{i}=\{(\right.$ Energy, 450) $\} \sqsubset \mathcal{R})$. However, if the resource summary

${ }^{9}$ That is, at compile time. 
procedure generateTree(Goal G, PlanLibrary P)

GoalNode $G:=$ new GoalNode $(\mathrm{G})$

$\operatorname{setTreeRoot}(G)$

$\operatorname{addChildren}(G, \mathrm{P})$

procedure setTreeRoot(GoalNode $G$ )

This procedure sets the goal $G$ as the root node of the goal-plan tree.

procedure addChildren(Node $G$, PlanLibrary P)

selectedPlans $(S P):=\operatorname{selectPlans}(G, \mathrm{P})$

addToGoalNode $(G, S P)$

for each $p \in S P$

$\operatorname{subGoalTree}(S G T):=\operatorname{extractSubGoals}(p)$

if $S G T==$ empty then

else $\operatorname{addToPlanNode}(p, S G T)$

for each $s g \in S G T$ addChildren $(s g, \mathrm{P})$ $\operatorname{attachSummary}\left(p,\left(P^{\mathcal{R}}(p) \oplus \mathcal{S}^{\prime}(S G T)\right)\right)$ $\operatorname{attachSummary}\left(G, \mathcal{S}^{\prime \prime}(G)\right)$

function selectPlans(Goal $G$, PlanLibrary P) returns PlanSet $P$ selects and returns the set of plans that satisfy the goal $G$

function extractSubGoals $(\operatorname{Plan} p)$ returns Node $n \in$ \{goal-node, sequence-operator, parallel-operator\}

Extracts the sub-goals from the plan body of $p$.

If the plan has no sub-goals this procedure returns an empty tree (null in programming terms).

If it has only one sub-goal, a node is created for that sub-goal (i.e. a goal-node) and returned.

If the plan has more than one sub-goal, these sub-goals will be either in parallel, sequential, or in a nested combination of the two. Hence, this procedure would create and return a tree structure similar to figure 4, with the root node being either a sequence-operator or a parallel-operator.

procedure addToGoalNode(GoalNode $n$, PlanSet $C$ )

adds each plan of plan set $C$ as a child node to the goal node $n$ in the tree.

procedure addToPlanNode(PlanNode $n$, Node $s g t$ )

adds the node $s g t$ as a child node of $n$. Note that $s g t$ is

either a single (sub) goal node or the head of a sub-goal tree.

procedure attachSummary(Node $n$, Summary $S$ )

attaches the summary $S$ to node $n$ in the tree

function $\mathcal{S}^{\prime}$ (Node $S G T$ ) returns Summary $S$

if $S G T=$ GoalNode then

return $(S G T)$.summary

if $S G T=$ SequenceOperator (;) then

return $\bigotimes_{\text {node } \in \text { children_of_SGT }} \mathcal{S}^{\prime}$ (node)

if $S G T=$ ParallelOperator $(\|)$ then

return $\bigoplus_{\text {node } \in \text { children_of_SGT }} \mathcal{S}^{\prime}($ node $)$

\footnotetext{
function $\mathcal{S}^{\prime \prime}$ (GoalNode G) : Summary $S$ then

return $\biguplus_{p \in \text { goalnode.children }} p$.summary
}

Fig. 3 Algorithms for deriving the goal-plan tree with resource summaries (see also Fig. 4) 
Fig. 4 A tree of sub-goals, which states that $\mathrm{G} 1$ is first executed followed by G2. After G2 completes, G3 and G4 are to be executed in parallel

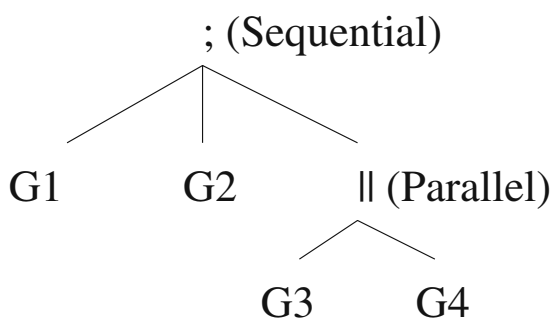

was not updated, then by using the original resource summaries the agent cannot deduce that it is safe to pursue the new goal $\left(\oplus P_{i}=\{(\right.$ Energy, 500) $\} \not \subset \mathcal{R})$ which is not desirable.

Note that the resource summary of each goal and its relevant plans are attached to the nodes of the goal-plan tree of the goal and that a template for this tree along with the resource summaries are created at compile time. At run-time each goal instance is assigned an instance of the associated goal-plan tree template. This tree

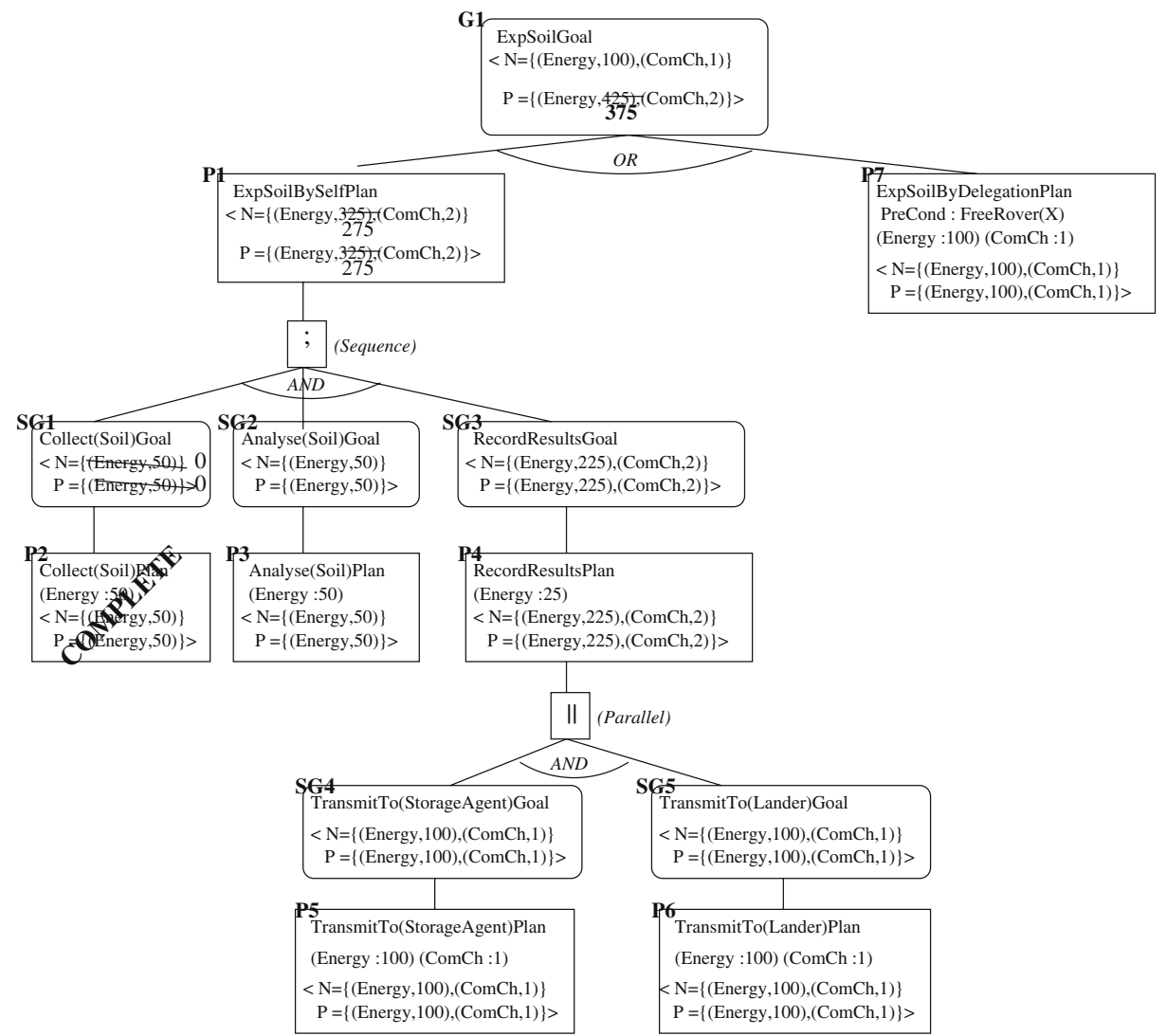

Fig. 5 Updated goal-plan tree for goal ExpSoilGoal 
can be updated at run-time by deleting nodes representing plans and sub-goals as they complete, and recalculating resource summaries each time a node is deleted.

Although only the path from the root to the modified portion of the tree needs to be traversed for each update, this may be costly if done routinely (depending on the number of goals being pursued in parallel and the branching factor of the tree). This can be easily optimised by flagging the nodes as requiring update, but delaying the actual propagation of changes to resource summaries until there is some known need for monitoring or recalculation. This recalculation occurs when the agent considers adopting a new potentially non-safe goal.

An additional simple optimisation which we use, is to maintain a count at each node of the number of children that use each resource. If the node is a plan node whose $\mathcal{P}^{R}$ requires a resource $t$, then the count for $t$ is 1 plus the number of children that require $t$. As a child node completes, this count is decremented. For example, in Fig. 5, at node $G_{1}$ the count for the resource Energy would be 6 initially (as there are six plans under the node that use it), but when plan $P 2$ completes, the count will be decremented to 5. When the count for a particular resource reaches zero, the need for that resource can be set to zero. In some cases this can allow faster updating and propagation, as once a resource need is zero or $P^{\mathcal{R}}$, remaining child nodes can be disregarded.

With these optimisations any cost beyond a couple of trivial operations is incurred only when a need is identified. The algorithm delete presented below, incorporates the above optimisations and provide a mechanism for updating the resource tree dynamically as plans and (sub) goals complete.

Updating the resource summary of a node (by the procedure update), sets the new value of the summary to the node and marks the parent of the node as dirty, which means that the parent's resource summary needs to be recomputed before use. This change is propagated all the way up to the root (using the procedure propogateDirty). The update procedure also decrements the usage count of the parent node (by the procedure decrementCount) and if the resource is no longer in use (i.e. usage count $=0$ ) then the summary for that resource is set to zero. It is not indicated as dirty, as it is not necessary to recompute that resource type, since it is no longer in use.

When resource summaries are relied upon (for instance to detect conflicts), if the resources of concern are dirty then they need to be recomputed before use. The recompute procedure does this. We assume that Summary $(t)$ returns a pair of records (necessary and possible) for the resource type $t$. For example, for goal $G_{1}$ in Fig. 5, summary $($ Energy $)=\langle 100,375\rangle_{6}$. Note that the subscript 6 in the notation denotes the usage count for that resource at goal $G_{1}$; there are six plans under that goal which require Energy.

Finally, recall that we assume that resource summaries are normalised. The algorithm for updating the goal-plan tree with resource summaries is shown in Fig. 6.

\subsubsection{Detecting Resource Conflicts}

The resource summaries in the goal-plan tree are maintained at run-time as goals execute, providing current information on the resources required for each goal to complete. We compare these up-to-date resource summaries of existing goals against the resource summaries of new goals to determine whether there will be possible or inevitable resource conflicts. 
procedure delete(node)

update(node, $\langle\emptyset, \emptyset\rangle$ )

if node $\neq$ root then $/ /$ remove the node from the parent's child list node.parent.children $:=$ node.parent.children $\backslash$ node

procedure update(node,newSummary)

if newSummary $=$ node.summary then $/ /$ no changes needed return

for each $t \in$ resourcetypes

$\left(n_{\text {old }}, p_{\text {old }}\right)_{\text {count }}:=$ node. $\operatorname{summary}(t)$

$\left(n_{\text {new }}, p_{\text {new }}\right):=$ newSummary $(t)$

if $\left(n_{\text {old }} \neq n_{\text {new }} \vee p_{\text {old }} \neq p_{\text {new }}\right) \wedge$ node $\neq$ root then

if $n_{\text {new }}=p_{\text {new }}=0$ then $/ /$ resource no longer needed decrementCount(node.parent, $t$ )

else if node $\neq$ root node.parent.dirty $[t]:=$ true propagateDirty (node.parent, $t$ )

node.summary $:=$ newSummary

procedure propagateDirty(node, $t$ )

if node $\neq$ root $\wedge$ !node.parent.dirty $[t]$ then node.parent.dirty $[\mathrm{t}]:=$ true propagateDirty(node.parent, t)

procedure recompute(node)

if $\neg \exists t$ such that node.dirty $[t]=$ true then return // as there are no nodes that are dirty

for each $c \in$ node.children recompute $(c) / /$ if there are child nodes recompute them first

if node is a GoalNode then node.summary $:=\biguplus_{c \in \text { node.children }}$ c.summary

if node is a PlanNode then node.summary $:=$ node.plan. $P^{\mathcal{R}} \oplus$ node.child.summary

if node is a ParallelOperator node $(\|)$ then node.summary $:=\bigoplus_{c \in \text { node.children }}$ c.summary

if node is a SequenceOperator node (;) then node.summary : $=\bigotimes_{c \in \text { node.children }} c$.summary

for each $t$ such that node. $\operatorname{dirty}[t]=$ true node.dirty $[t]:=$ false // updated hence not dirty anymore

procedure decrementCount(node, $\mathrm{t}$ )

$(n, p)_{c}:=$ node.summary $(\mathrm{t})$

summary $(\mathrm{t}):=(n, p)_{c-1}$

if $(c-1)=0$ then $/ /$ resource no longer in use summary $(\mathrm{t}):=(0,0)_{0}$

if node $\neq$ root then decrementCount(node.parent, $t$ )

else

node.dirty $[\mathrm{t}]:=$ true

if node $\neq$ root then

propogateDirty(node.parent, $t$ )

Fig. 6 Algorithm for updating the goal-plan tree summaries at run-time 
A set of goals can be determined to be in one of four categories:

- Safe-if the available resources are sufficient to satisfy the combined ${ }^{10}$ possible resource requirements of all the goals. They can be safely executed in parallel.

- Conflicting - if the available resources are insufficient to meet the combined necessary resource requirements of the goals then there is an inevitable resource conflict. Some goals will have to be rejected or wait for more resources to be made available. Note that while necessary does not imply sufficient, it provides a lower bound that allows a definite conflict to be determined.

- Schedulable-due to insufficient resources that are of type re-usable some goals may conflict if executed in parallel. These conflicts may be avoided if the goals are interleaved appropriately.

- Uncertain-when a determination of safe, conflicting, or schedulable cannot be made. That is, when the resources available are greater than the combined necessary resource requirements but less than the combined possible resource requirements of the goals. In this situation the agent can be cautious and reject the new goals or allow the goals to be pursued, monitoring them until they can be deemed to be in one of the above categories and an appropriate action taken.

\subsection{Avoiding Interference}

When two parallel goals are interleaved, the steps taken to achieve one goal may interfere with a sequence of steps required to achieve the other goal. For example, suppose a Mars rover agent has a goal to analyse soil samples at a location $A$. Amongst others, two of the steps to achieve this goal would be to go to location $A$ and collect soil sample in that order, with the effect $A t A$ achieved by the first step being a pre-condition to the other. In this case, having arrived at location $A$, a rational agent should not allow a plan which took the agent elsewhere to intervene, prior to the agent collecting a soil sample.

There are two important ways in which parallel goals can interfere, which we try to capture and reason about. Considering the logical outcomes of goals and plans, interference can occur:

- When an in-condition is made false while a plan or goal is executing, causing the plan or goal to fail.

- When a previously achieved effect is made false before a plan or goal that relies on it begins executing, preventing the goal or plan from being able to execute.

Recall that in-conditions are conditions that must remain true during the pursuit of a goal or execution of a plan. In order for a goal/plan to begin execution, the in-conditions (if any) must be true, and must not be undone until the goal/plan completes, as this would cause the goal/plan to fail. In pursuing their goals, intelligent agents often use a series of plan steps where the effect of an earlier step achieves the pre-condition for a later step. We call these effects preparatory-effects. A rational agent should not allow preparatory-effects which have been achieved to be interfered

${ }^{10}$ Algorithms for combining resource summaries were also presented in [25]. 
with, until the later step for which they are pre-conditions completes. Pre-conditions that are not preparatory-effects need not be protected as there is no clear justification for protecting them.

The summary information that must be maintained at each node of the goal-plan tree for reasoning about interference includes:

- Effects (as they could cause the interference);

- In-conditions; and

- Preparatory-effects (as these need to be protected when active).

Each of these are categorised into definite and potential where;

- A definite condition/effect will definitely be achieved/required (achieved for effects and required for in-conditions) at some point along every possible path of achieving the goal (i.e. this condition need not be required by all plans, but is required by at least one plan in every possible way of achieving the goal); and

- A potential condition will possibly be achieved/required at some point in the pursuit of the goal. This condition is part of at least one plan in at least one path of achieving the goal, but is not achieved/required in all possible paths of achieving the goal.

Unlike resource summaries where necessary resources were a subset of the possible resources, definite and potential conditions/effects are defined to be exclusive of each other.

In addition to this summary information each plan node also contains dependency information which identifies any plans or sub-goals that depend on an effect (preparatory-effect) of the plan.

As with resource summaries, the summary information and the dependency entries are created at compile time for each goal-plan tree and updated at runtime for each instance of it.

Our algorithms indicate for each new goal being considered, whether it would:

- Definitely not cause any interference-It is safe to pursue the goal in parallel with existing goals.

- Definitely cause interference-Then either the goal is not pursued or existing goals that interfere with it are preempted, depending on the agent's preference.

- Possibly cause some interference-When the interference is potential the goals are pursued in parallel, but monitored for steps which cause conflict, and if necessary suspended so that interfering steps wait until the steps that they interfere with are complete.

\subsection{Facilitating Positive Interactions}

We have discussed two kinds of potential negative interaction between concurrently executing goals. However the following is an example where two goals can interact positively:

Consider a Mars rover agent that has a goal of analysing soil samples at location $A$ and a goal of analysing rock samples also at location $A$. The agent could pursue these goals sequentially by going to $A$, analysing a soil sample, returning back to the lander, then once again going to $A$, analysing a rock sample and returning back to the 
lander. Alternatively it could go to $A$, analyse a soil sample, analyse a rock sample and then return to the lander. Clearly the second approach is more intelligent, and is brought about by the agent reasoning about the common steps between the two goals and deducing if these common steps can be combined (joined) for the two goals. While this reasoning is not necessary for the agent to perform its tasks correctly, it can make the agent more efficient. For example, in situations where resources are limited, combining plans may assist in more goals being achieved. In the Mars rover example above, the agent will save fuel by making a single trip to location $A$, instead of two.

In order to provide a framework to allow the agent to detect and facilitate such positive interactions, we again use the goal-plan tree structure. We extend the effect summaries used for reasoning about interference to include information about the particular plan instance which is intended to achieve the effects (at runtime). This assists in scheduling plans when common effects exist. As previously, most of the summary information is calculated at compile-time. however, at runtime a handle to the relevant plan instance is stored.

The algorithms check for cases where steps of two goals that bring about the same effect could possibly be combined (joined). These steps of a goal, in BDI agents, are sub-goals. Our algorithm goes beyond simply recognizing multiple occurrences of the same sub-goal step, but also looks for any types of sub-goals that bring about the same effects. These sub-goals are achieved by plans, and when we join sub-goals, we are essentially joining the plans that achieve them.

Once we have identified effects that are common to plans of different goals, we maintain a record of the plans that achieve each common effect. We differentiate between plans (with common effects) that will definitely be reached (executed) by the goals concerned, and those that may only potentially be reached; as there is a greater chance of joining plans that are definite rather than those that are potential.

Data structures are used to monitor for when one plan that has a common effect is reached, so that it can be temporarily suspended, until the other plans, that also achieve the same common effect as the suspended plan, are reached. When all the plans for a common effect are synchronized, they can be joined (under certain conditions) resulting in just one of them being executed.

When synchronizing plans, it is possible for deadlocks to occur, but we have incorporated steps for preventing these.

When the agent decides to pursue a new goal, the effect summary of the new goal is compared with the effect summaries of current goals and commonalities are registered. There are three cases of two plans having a common effect. The common effect may be:

- Definite to both plans-In this case plan scheduling is performed to facilitate the join.

- Potential to both plans-Given that neither plan is certain of being reached the agent can either be optimistic and schedule plans hoping the plans in subject are chosen, or be cautious and not attempt a plan join.

- Definite to one plan, but potential to the other-In this case if the potential plan is reached first, then the agent will suspend that plan and wait for the definite plan to reach and perform a join. However, if the definite plan is reached first, then since the other plan is only potential, the agent can choose to be either cautious or optimistic. 
If the type of application is more resource critical than time constrained, then the optimistic approach would suit better; since it will try to join plans in all possible situations. However, it may waste time. Therefore, if the application is more timeconstrained then the cautious approach would suit better as it would ensure that the agent would not unnecessarily delay the execution of its plans for a join that may never take place. In our experiments below we assume a cautious agent.

\section{X-JACK}

We extended JACK Intelligent Agents ${ }^{\mathrm{TM}}$ [5], a Java based industrial BDI system to incorporate our data structures and reasoning algorithms about managing interactions between goals. We call this extension $X$-JACK.

There is no explicit representation of goals in JACK. Goals are implicitly represented as events which trigger the execution of plans. We extended this by introducing an explicit structure for goals, a goal template, that includes the attributes of GoalTypeName, Plans, In-Conditions and Effects as described in the previous section.

A JACK plan consists of:

- A relevance condition, which determines if the plan is relevant to a particular event;

- A context condition (pre-condition), that determines the applicability of the plan given the current state of the agent;

- A plan body, which can be any combination of sub-goals and arbitrary Java instructions; and

- pass and fail methods, that are called when the plan succeeds or fails respectively.

In addition to the above, in X-JACK, to each plan we:

- Attached the resource requirements of the plan (excluding the resource requirements of its sub-goals);

- Attached the effects of the plan (excluding the effects of its sub-goals);

- Attached the in-conditions of the plan (excluding the in-conditions of its subgoals); and

- Modified the relevance condition to check for relevance to a goal-type, rather than an event.

In order to manage the pursuit of goals we incorporated a Goal Management System (GMS) into X-JACK. Goals are added to the agent system via the GMS. At compile time, the GMS creates a goal-plan tree template for each goal-type. When a goal is added to the GMS at run-time, a goal instance is created and a goal-plan tree instance of the appropriate goal-type is attached. The GMS uses the reasoning methods developed in previous work $[23,25]$ to identify whether the new goal is safe to be pursued with existing goals, or if monitoring is required. If the goal is safe, the GMS will allow the goal to be pursued using the normal JACK routine of plan selection and execution. If the goal is not safe, the appropriate steps as determined by our reasoning methods will be followed. When goals are safe to be pursued, the GMS will also monitor for positive interactions and facilitate any identified, using the algorithms that we have developed in [24]. 
In order to facilitate the process of dynamically updating goal-plan tree instances, and for monitoring and scheduling plans, we modified:

- The plan body such that as soon as a plan is activated it registers itself with the GMS and waits until the GMS allows it to be executed; and

- The pass and fail methods of plans, such that the GMS is notified when a plan succeeds or fails respectively.

This extension also allows the GMS to monitor the progress of each goal which, although not utilised in this work, is a useful feature in an agent system.

\section{Evaluation}

In order to assess the efficiency and the effectiveness of our modifications to JACK, we ran a series of experiments to empirically evaluate the three different algorithms under a range of conditions.

All the evaluation experiments were run on an Intel Pentium 4, dedicated PC, under Linux 7.2 operating system. The speed of the processor was $1.7 \mathrm{GHz}$, and $512 \mathrm{MB}$ of RAM were available. The scenarios were developed using JACK version 4.1 and Java version 1.4.2.

On the results obtained, when required, tests for significance (p-value tests) were done by performing the standard Student's t-test on the relevant data sets using PHStat Statistical Add-In for Microsoft Excel, version 1.4.

Where no variance was found in the data population, the p-value was determined by performing the Wilcoxon rank sum test which is a non-parametric equivalent of the unpaired t-test, also using PHStat.

We describe separately the set of experiments run on each algorithm, and the results obtained.

\subsection{Resource Conflict Management}

There is no specialised mechanism for representing resources and simulating resource usage in JACK. Therefore, for comparison purposes, we allowed resource usage to be specified at the plan levels in JACK, as we did in X-JACK. In order to simulate resource usage we introduced a resource manager, which we implemented identically in both JACK and X-JACK. The resource manager merely simulates resource usage and does not perform any conflict management, which was done only by the GMS in X-JACK and was not present in JACK.

In designing our experiments we attempted to identify situations which would make heavy use of the new data structures and algorithms in order to test them under extreme circumstances. We also identified characteristics that could be observed about applications, such as the extent to which plans tend to use the same resources, or the extent to which resources tend to be insufficient to achieve all goals. The characteristics we chose to focus on were as follows:

1. The number of goals that run in parallel. The more goals that run in parallel, the more they interact with each other (if they compete for resources). This allows us to test the cost of the reasoning as the number of goals increases. This characteristic also enables us to test the benefits of the system since greater 
interaction also means greater chance of conflicts, which, if detected early can be avoided. We controlled the number of goals that ran in parallel by varying the delay in adding goals into the system.

2. The extent to which goals require the same resources. The greater the number of goals that compete for a single resource, the greater the chance for conflicts to occur. A situation with heavy use of the same few resource types provided a stress-test for the algorithms, as it increased the size of the data structures and the number of iterations within the algorithms. A high level of conflict also provided opportunities to benefit from recognition of that conflict. This variable therefore was expected to affect both costs and benefits.

3. The amount of resources available. Limited resource availability also contributes to greater conflict between goals, and therefore opportunities to benefit from early detection of conflict.

4. The total number of steps that get executed for a single goal. If a goal has many steps, there is a greater chance that it will waste resources doing early steps, before failing due to lack of resources at a later step. Thus an application with goals that require a number of steps is likely to benefit more from early conflict detection, than one where goals are accomplished in very few steps. Also, the size of the data structures grows as the number of goals and plans increase which will affect the run-time cost of the algorithms. The number of steps, or number of plans executed, arise from both the depth of the goal/plan hierarchy and the number of steps (sub-goals) within a plan.

5. The depth of the goal/plan hierarchy. This affects the total number of plans executed, but in addition it affects the amount of updating of resources required at run-time as the goal executes.

6. The type of the resource. Reasoning about conflict with re-usable resources needs to be evaluated separately from reasoning about consumable resources, as whilst conflict can be avoided by appropriately scheduling of re-usable resources, scheduling will not resolve conflicts due to consumable resources.

In order to compare the behaviour of X-JACK (incorporating our additional reasoning) with JACK, we set up abstract scenarios varying the characteristics described above. The experiments used two different structures of top level goals, as shown in Fig. 7. These are referred to as "depth 2 goals" and "depth 5 goals". These goal structures capture both differences in plan numbers and differences in goal depth. The percentage of goals vying for the same resource was varied, as was the total availability of resources. The runtime for a single goal of depth 2 was approximately $62 \mathrm{~s},{ }^{11}$ and for depth 5 was $162 \mathrm{~s}$. The amount of parallelism was varied by changing the rates at which goals were added into the system (the shorter the interval, the greater the parallelism and vice versa).

Each experiment ran 50 goals in total, and was run 20 times, with the ordering of goals chosen randomly. In the following experiments, the time recorded was the actual clock time taken for the experiments to complete.

\footnotetext{
${ }^{11}$ Artificial waiting was introduced into plans, to simulate real actions taking time. The waiting time was $20 \mathrm{~s}+/-0-10 \%$ which was randomly generated for each plan. However, the random seed was the same for multiple runs ensuring that the multiple runs were conducted equally. While the plan was waiting no other plan was in execution.
} 


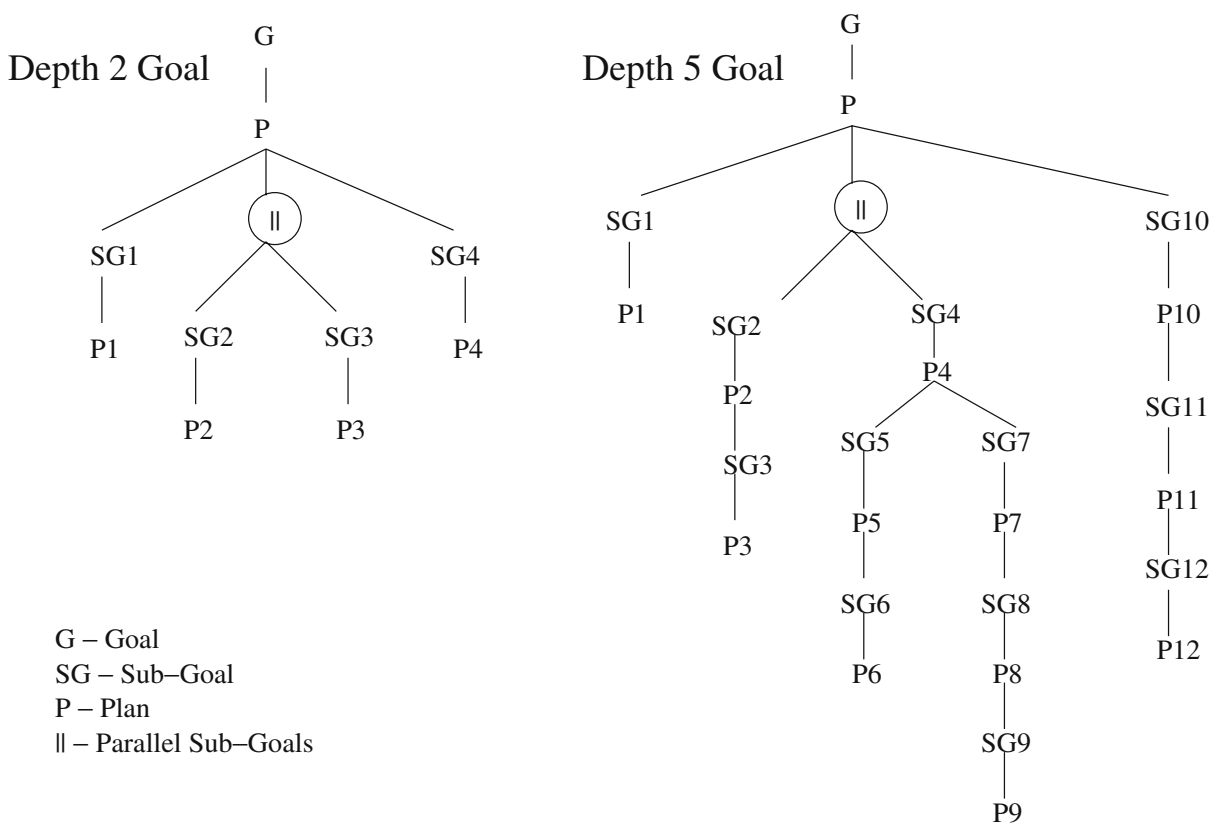

Fig. 7 Top level goal structures, as used in the experiments

This set of experiments can be grouped into 4 different explorations:

1. Costs and benefits at differing levels of resource availability, with high levels of interaction between goals;

2. Costs and benefits at differing levels of resource availability, with normal/lower levels of interaction between goals;

3. Costs and benefits at differing levels of resource availability, with different types of resources (i.e. re-usable and consumable); and

4. Computational cost of reasoning about resource conflicts.

The run-time cost of reasoning was measured by recording the time taken for all goals to complete (either by success or failure). The benefit was measured by the number of goals that completed successfully (out of the 50 that were pursued).

\subsection{High Levels of Goal Interaction}

To evaluate the algorithms in situations where there is a high level of interaction between goals, the following steps were followed:

- A scenario was set up, where each of five consumable resource types were used by $20 \%$ of the goals. Each goal used two resource types, and these were chosen to ensure high interaction-e.g. goal type 1 used resource types $\mathrm{A}$ and $\mathrm{B}$, goal type two used resource types $\mathrm{B}$ and $\mathrm{C}$, and so on.

- Resource availability was then varied to range from $25 \%$ to $100 \%$ of that required for all goals to complete. 
- Separate scenarios were set up for 50 goals of depth 2 and 50 goals of depth 5 (refer to Fig. 7 for example of goal depths).

- Goals were chosen at random from the 50 available, and added to the system at a set interval. Initial experiments were run with an interval of $2 \mathrm{~s}$, and the experiments were re-run for an interval of $20 \mathrm{~s}$. The fastest rate of addition allowed up to 30 goals of depth 2 or 50 goals of depth 5 to have some of their execution time in parallel. The slowest rate allowed parallelism between three goals of depth 2 and eight goals of depth 5.

Figures 8 and 9 , show the average timings and goal completions, where goals were added every $2 \mathrm{~s}$, and every $20 \mathrm{~s}$ respectively, with varying resource availability.

The situation with goal depth 5 and addition of goals every $2 \mathrm{~s}$ (Fig. 8), required most processing from X-JACK, and also realised the most benefit. Under these extreme circumstances, even at $95 \%$ resource availability, there was a clear and significant benefit in using X-JACK, in terms of successful goal completions. In terms of run-time cost it can be seen that X-JACK takes more time for each run. However, it is not possible to ascertain here how much of the extra time by X-JACK was due to successfully executing more goals to completion, and how much of the extra time is spent on reasoning about conflicts. We leave this analysis to a later experiment in this section.

The situation with goal depth 2 and addition of goals every $20 \mathrm{~s}$ (Fig. 9), is the situation (in this experimental set) which offered least opportunity for benefit, but

Depth 2
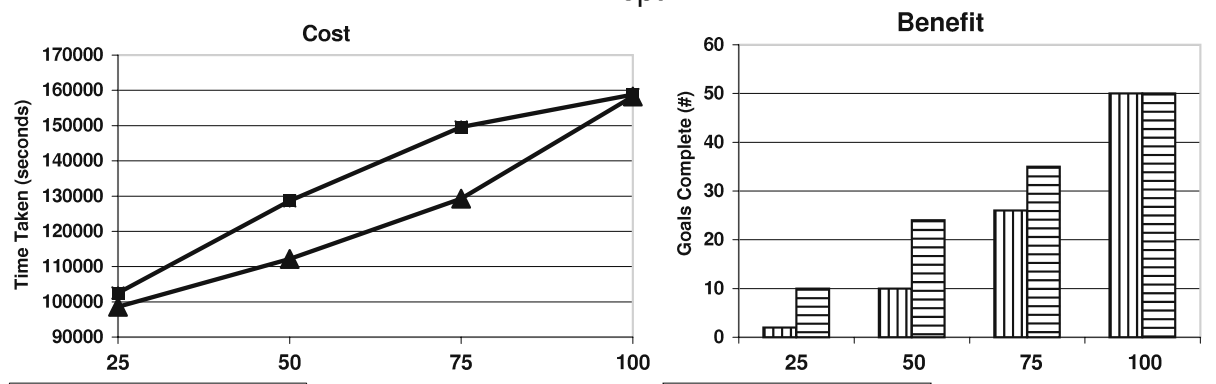

- J JACK $\rightarrow$-X-JACK Resource Availability (\%) DJACK 曰X-JACK Resource Availability (\%)

Depth 5
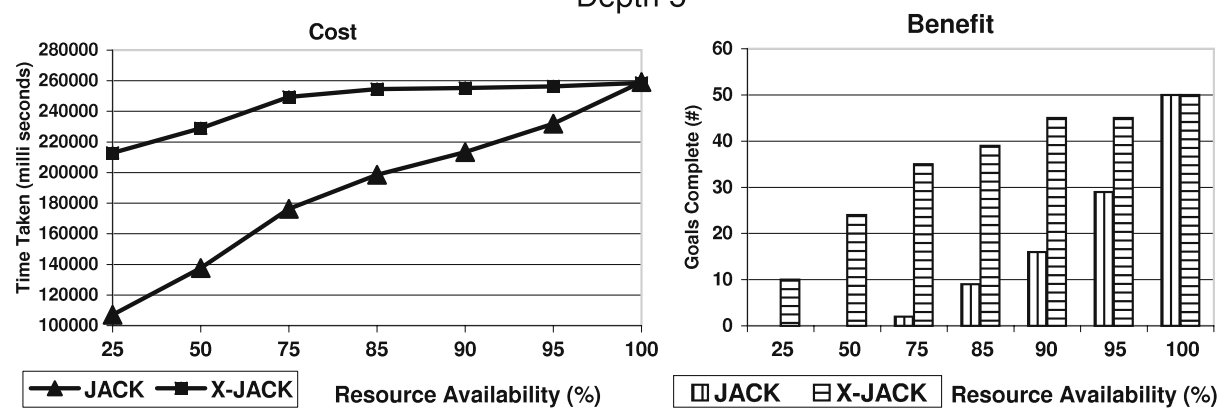

Fig. 8 Resource conflicts: high levels of goal interaction, with delay of $2 \mathrm{~s}$, and high parallelism 

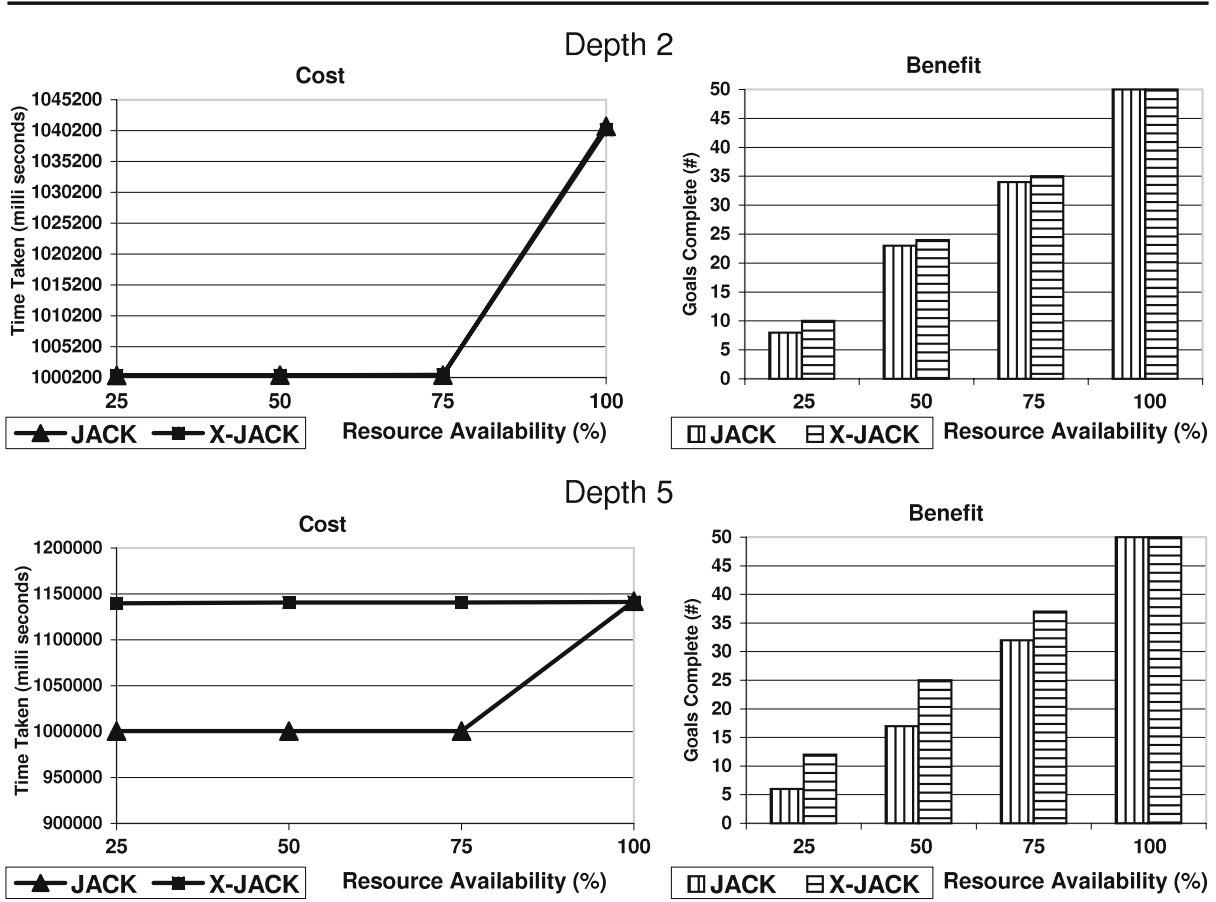

Fig. 9 Resource conflicts: high levels of goal interaction, with delay of $20 \mathrm{~s}$, and low parallelism

also required least additional processing. There was still a slight advantage in using X-JACK up to $75 \%$ of resource availability. This benefit was still statistically significant, with an observed level of significance (p-value) of 0.049. Differences in time taken (i.e. run-time costs) were not statistically significant at the $\mathrm{p}<0.05$ level with p-values of $0.28,0.68,0.71$ and 0.83 for resource availabilities $25 \%, 50 \%, 75 \%$ and $100 \%$ respectively.

In summary, the lower the resource levels, the higher the number of goals running in parallel and the greater the amount of plans required to complete by each goal, the greater the benefit is in using X-JACK. Even in situations of least benefit, other than where there are adequate resources to avoid conflict, X-JACK shows small yet significant benefits over JACK.

\subsection{Lower/Normal Levels of Goal Interaction}

In these experiments, we set up two scenarios (using depth 2 goals and depth 5 goals) where 40 of the 50 goals had no resource interactions (used no resources) and the other 10 goals each used five consumable resource types. Each of the five resource types was used by $20 \%$ (i.e. two) of the goals, and each goal used two resource types. This was expected to lead to a more realistic rate of conflict over resources, and therefore give a better view of potential benefits.

Figure 10 shows the results of these experiments. We note that even in this more realistic situation, X-JACK showed benefits in goal completions as compared to JACK, when there were insufficient resources for all goals to complete. The p-value 

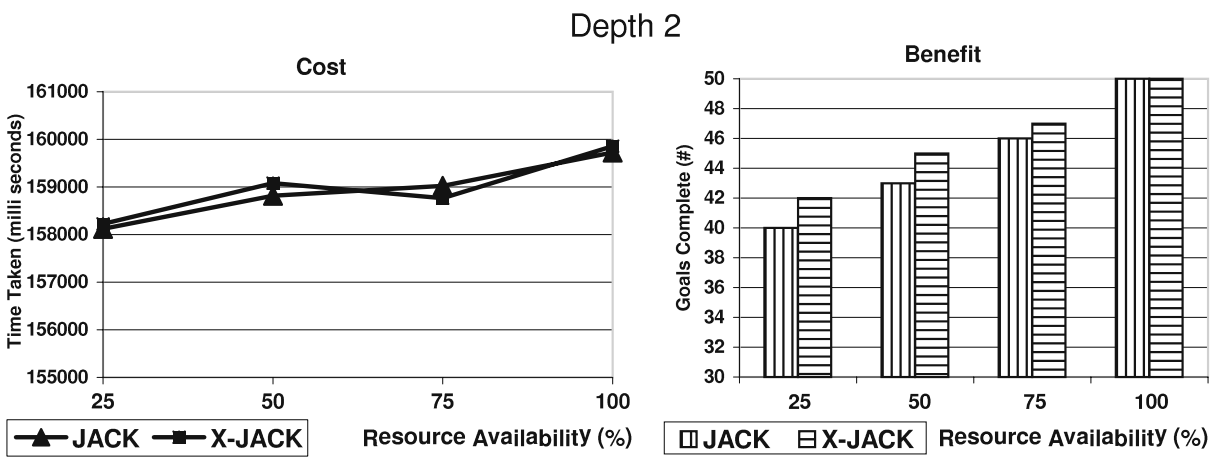

Depth 5
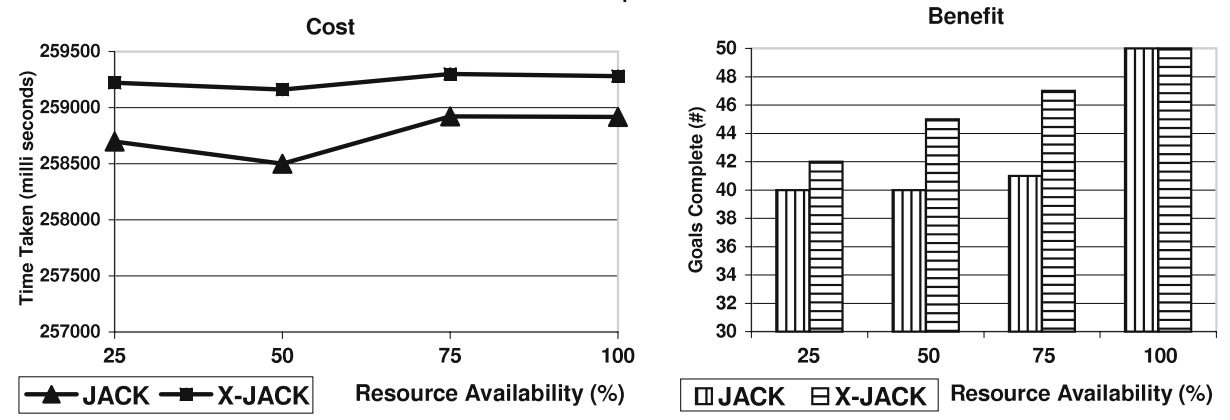

Fig. 10 Resource conflicts: results with lower levels of goal interaction

for the difference in the number of goals that successfully completed was less than 0.00016 for $25 \%, 50 \%$ and $75 \%$ of resource levels, showing that the improvement in percentage of goals achieved was significant.

There was a noticeable increase in time required for X-JACK to run. However, with the exception of $50 \%$ resource availability for depth $5(\mathrm{p}=0.018)$, differences were not statistically significant at the $\mathrm{p}<0.05$ level (actual values were $0.279,0.488$ and 0.285 for resource availabilities $25 \%, 75 \%$ and $100 \%$, respectively).

Note again that it was not possible to determine from these experiments what proportion of the extra time was spent on reasoning and what proportion was spent on achieving goals which otherwise would have failed quickly.

\subsection{Re-usable Resources}

In order to compare the behaviour of JACK and X-JACK when the resources were of type re-usable, we used the same experimental setup as in Section 5.1, with the exception that the goals used five re-usable resource types instead of consumable. The results obtained are shown in Fig. 11. In this situation X-JACK completed all 50 goals irrespective of the percentage of resources available. This was because the plans which required the re-usable resources were scheduled to avoid conflict. This scheduling could however cause some run-time delay as we observed in the case of $25 \%$ resource availability for depth 5 . JACK performed the same as it did for consumable resources (refer Fig. 10). 
Depth 2
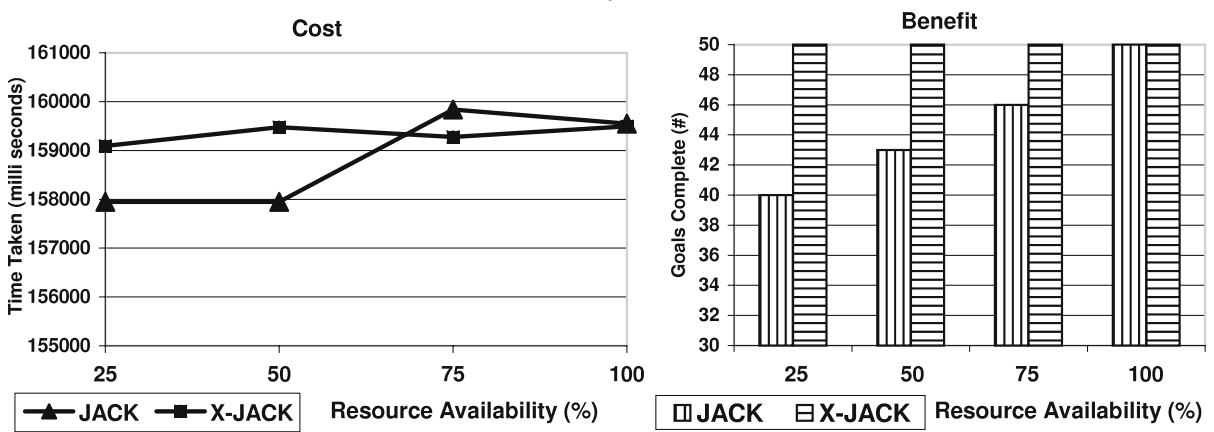

Depth 5
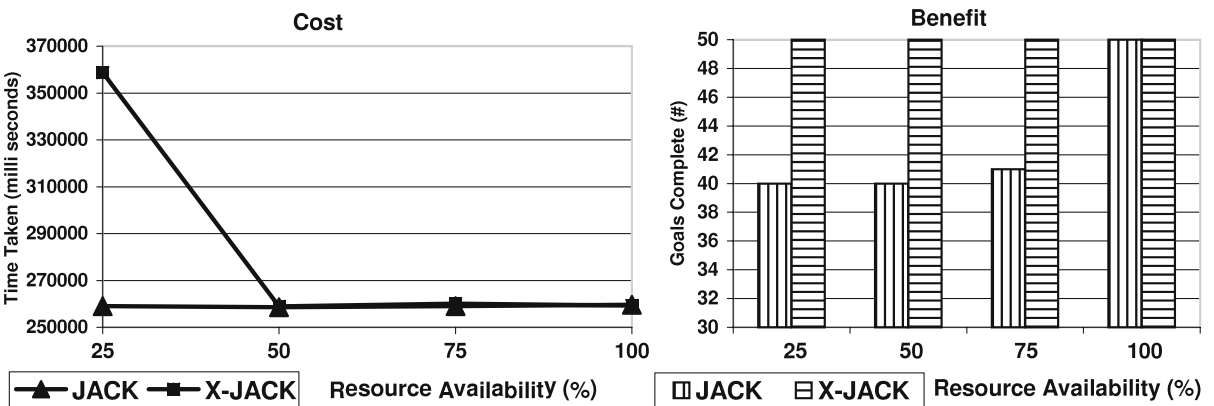

Fig. 11 Resource conflicts: results with re-usable type of resources and lower levels of goal interaction

These experiments show that while X-JACK shows benefit (in terms of goal completions) when the resources are of type consumable, the benefit is much greater for resources of type re-usable.

\subsection{Computational Cost of Reasoning About Resource Conflicts}

From the above experiments, it was not possible to ascertain the actual overhead of the additional reasoning, that is, the computational costs. This is because the two systems were successfully completing a different number of goals. In order to determine the approximate cost of the reasoning, we re-ran the experiments as described for high levels of resource conflicts, for depth 5 with the goal addition interval at $2 \mathrm{~s}$ (i.e. the most stressful situation), ensuring that the same goals that completed for X-JACK also completed for JACK. ${ }^{12}$ This gave us comparable timings for the same number of goals to run, without the additional reasoning. The results obtained are shown in Fig. 12.

We found that when there was no conflict (i.e. 50 goals complete), the reasoning overhead was insignificant (statistically, $\mathrm{p}$-value $=0.29$ ). Whilst there was a

${ }^{12}$ This was achieved by noting the goals that were successfully pursued in X-JACK and running these same goals in JACK. 
Fig. 12 Resource conflicts: computational cost
Depth 5, Resource Usage 20\%

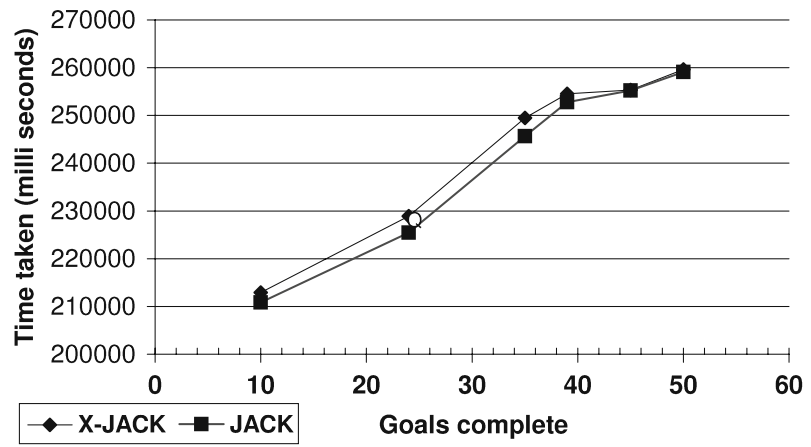

difference in the time taken when there were conflicts in the system, the difference was very low. For example, the situation where there were most conflicts was when 10 goals completed. The difference in the time taken between the two systems at this point was less than $1.8 \mathrm{~s}$. Given that JACK, with the same level of conflict, did not complete any goals (refer Fig. 8), an overhead of less than $1.8 \mathrm{~s}$ to complete 10 goals as opposed to none is certainly acceptable in most situations.

\subsection{Avoiding Interference}

Similar to the experimental work on resource conflicts, we developed abstract scenarios which stress-tested the algorithms, as well as scenarios that were more realistic in real world applications.

Some of the factors that contribute towards the complexity of the algorithms that avoid interference are the amount of interaction between goals, the number of goals that execute in parallel, and the duration of the plans executed to satisfy a goal. ${ }^{13}$ These can be explained in more detail as follows:

- The amount of interaction between goals. When more goals interact in terms of: (1) the effects that their plans bring about; (2) the in-conditions of both goals and plans; and (3) the preparatory effects set up by plans, there is a greater chance of interference. Greater interference may result in a greater number of goals failing, if the interference is not managed. High interference would also stress-test the algorithms computationally. So we expect that high interference would result in greater benefit but may also incur a higher cost.

- The number of goals that execute in parallel. As more goals execute in parallel, there is a greater chance of interference between goals. Therefore, there is likely to be a greater benefit from our algorithms as the amount of parallelism increases.

- $\quad$ The duration of the plans required to satisfy a goal. The longer the plans take to execute, the longer the active in-conditions and preparatory effects need to be protected, which increases the chance of interference.

\footnotetext{
${ }^{13}$ These were also factors with respect to resource conflicts as described in the previous section.
} 
Experiments were conducted to assess:

- $\quad$ Run-time costs and benefits of avoiding interference when there is a high level of interactions;

- Run-time costs and benefits of avoiding interference when there is a lower, more normal level of interactions;

- The effect that varying levels of parallelism have on interference between goals; and

- The computational cost of the algorithms that manage interference between goals.

In the experiments that we conducted the general setup was as follows. We created 10 different types of goals in terms of the goal/plan structure, and at runtime instantiated 20 goals whose type were randomly selected from those goal types.

We ran each experiment 50 times, and in each run selected a different random combination of goal instances. These runs were tested in JACK and X-JACK such that the 50 random combinations of goal instances were identical in both systems.

The time taken for all 20 goals to complete either by success or failure was recorded along with the number of goals that successfully completed. The former provides a run-time cost estimate while the latter indicates the benefit of using our mechanisms for avoiding interference.

\subsection{High Levels of Interference}

In order to stress-test our algorithms, we used the experimental setup as above, and set up the level of interactions such that each goal type interacted with another two goals which could potentially cause interference. This created an unrealistically high level of interactions between goals.

The results obtained in terms of the run-time cost and benefits are illustrated in Fig. 13. The figure shows that the benefit is very large; with JACK on average completing only half the amount of goals that X-JACK completes. There is however, a higher run-time cost in X-JACK, but that is expected since X-JACK executes more
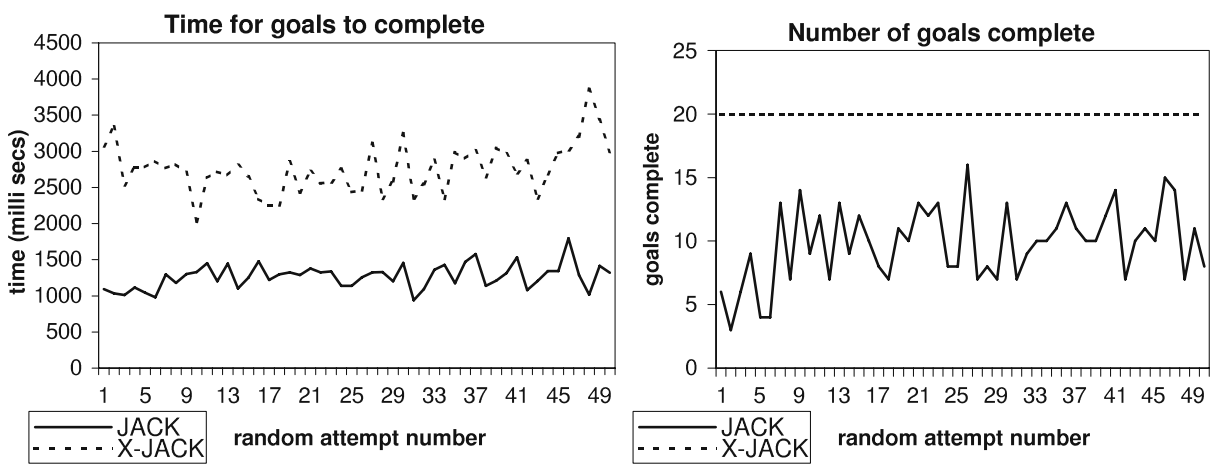

Fig. 13 Interference: results with high levels of interference 

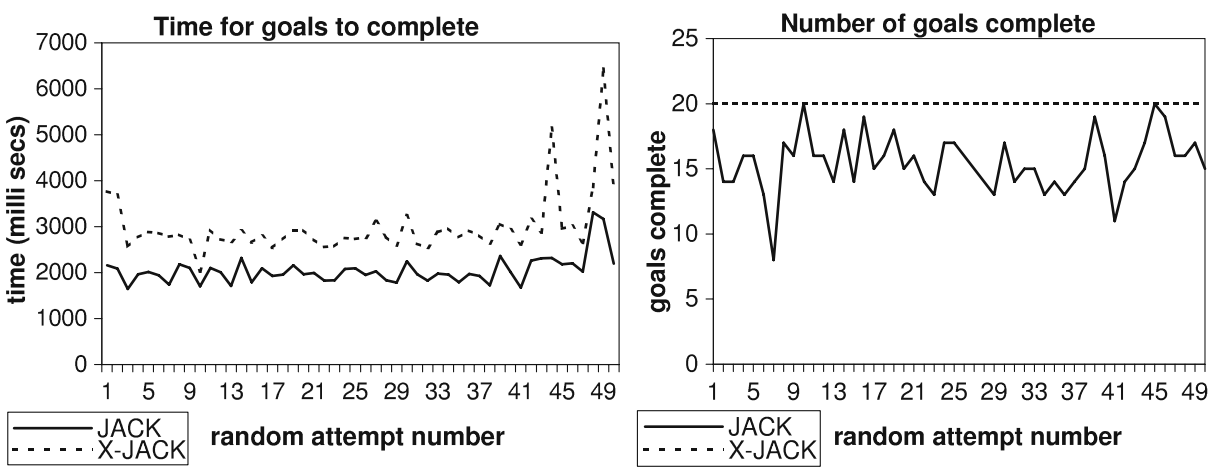

Fig. 14 Interference: results with lower/normal levels of interference

goals to completion than JACK, and also since when plans are suspended, they have to wait until the plans or goals that they conflict with complete.

\subsection{Lower/Normal Levels of Interference}

In order to ascertain the benefits in more realistic situations, we used the setup described above, but set the level of interference such that, four of the 10 goal types did not have any interactions with other goals, three of the goal types interacted with one other goal and the other three interacted with two other goals each.

The results obtained are shown in Fig. 14. We observe that the benefit is still quite evident, with JACK on average achieving five goals less than X-JACK. In terms of the run-time cost, X-JACK took significantly more time than JACK (statistically, $\mathrm{p}$-value $\left.=1 \times 10^{-17}\right)$. This additional time may have been caused by:

- X-JACK executing more plans as it successfully completed more goals than JACK. This is not caused by the algorithms that avoid conflict, and is not a disadvantage to the system, as it is the expected run-time cost of pursuing goals.

- Plans being suspended due to conflict, and waiting until it is safe to continue. This is caused by the reasoning algorithms in X-JACK. When plans are suspended in the event of potential conflict, the waiting time is unnecessary. However, if the plans are not suspended they may cause conflict, and as a result a lower number of goals will be achieved.

- The computational cost of the reasoning algorithms. This introduces an additional overhead into the system and it is important for the algorithms to have a low computational cost. However, we cannot ascertain this from this set of experiments and we leave it for a later set.

In order to identify the effect of having plans with longer durations, we increased the length of each plan to twice the original length ${ }^{14}$ and re-ran the experiments. The results that we observed are shown in Fig. 15. In this case, while we do not see much of an added benefit when plans are longer, the run-time costs has increased in

${ }^{14}$ Plan lengths were increased by increasing the simulated action times within the plan. 

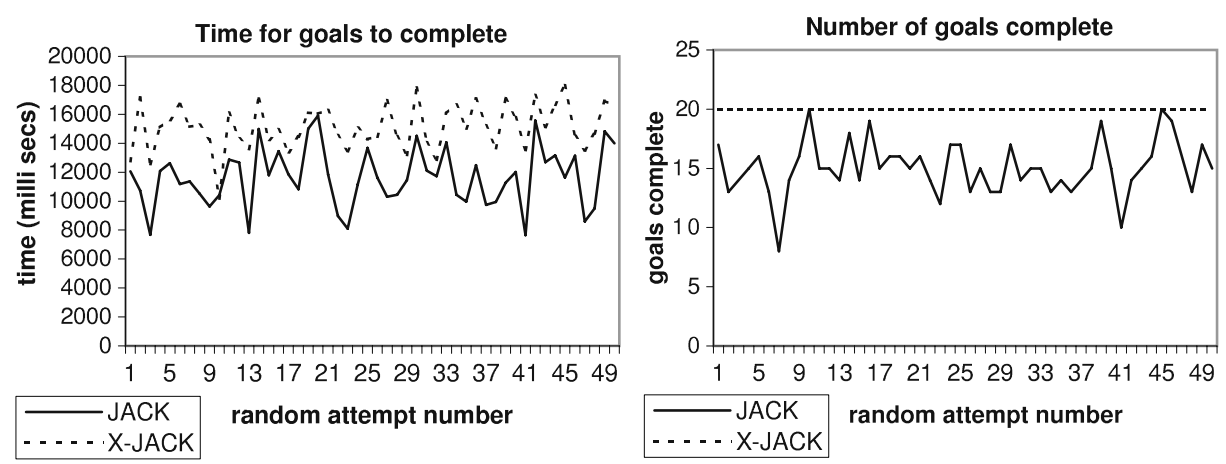

Fig. 15 Interference: results with longer plans

X-JACK. This increase was expected as goals and plans that are suspended would have to wait a longer time until the goals/plans with which they conflict completed.

\subsection{Varying Levels of Parallelism}

In this set of experiments we investigated how varying levels of parallelism between goals affect the level of interference between them. In order to do this, we used the same setup as for lower/normal levels of interactions above, but added the goals to the system via the GMS (Goal Management System) at different time intervals. The greater the time interval, the lower the chance was of the goals having steps executing in parallel.

Figure 16, shows the number of goals completed when the time interval of adding goals was 10, 25, 50 and a $100 \mathrm{~ms}$ long. From the figure we can see that as the time interval is increased (i.e. parallelism decreased), the lesser the conflict that occurs, and hence the lesser the benefit of X-JACK. However, the benefit is still significant

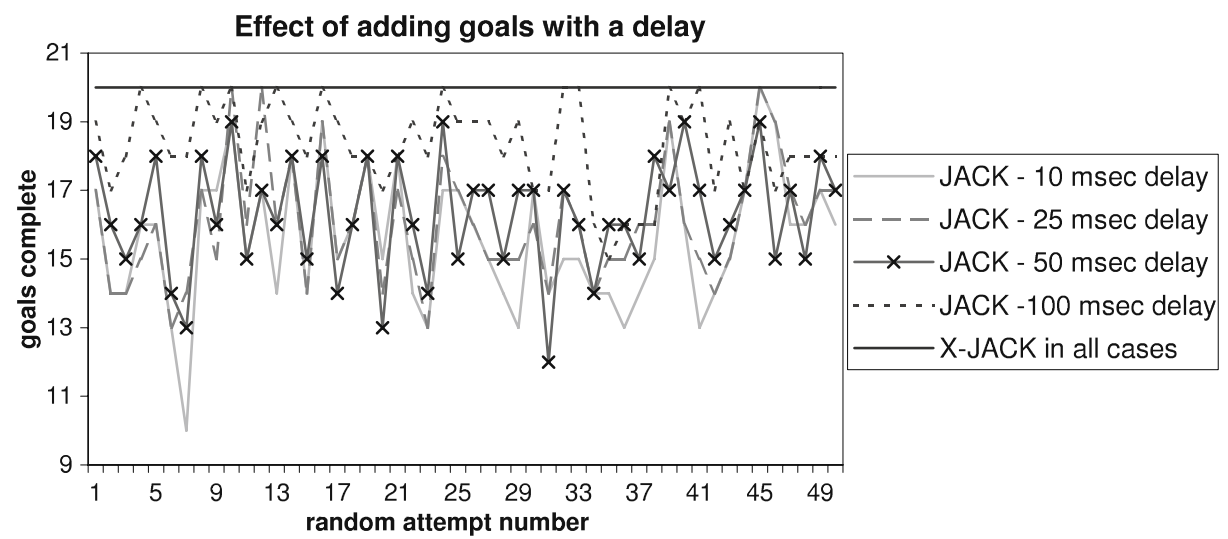

Fig. 16 Interference: effect of adding goals to the system at varying time intervals 


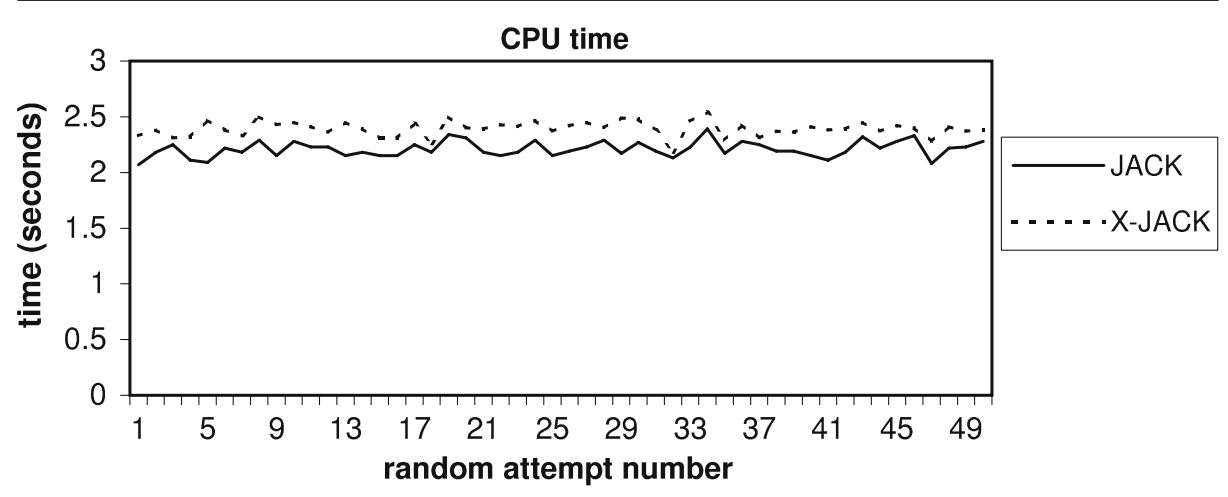

Fig. 17 Interference: computational cost

even when there are lower levels of parallelism, in that X-JACK always completes all the goals, but JACK only does so in $20 \%$ of the total runs.

\subsection{Computational Cost of Avoiding Interference}

In order to determine the computational cost of the reasoning algorithms, we took the situation where the algorithms were stress-tested, that is the setup for high levels of interference, and modified:

- JACK, such that plans would not fail, even if the pre-conditions and in-conditions were not met $^{15}$ (that is all 20 goals complete successfully); and

- X-JACK, such that even though the reasoning algorithms were executed, plans were not suspended and were allowed to continue, and as we did for JACK, plans would not fail even if the pre-conditions and in-conditions were not met. Although the actual plan suspension was not done in X-JACK, the process of identifying interference and synchronizing plans to avoid them was still done to reflect the true computation involved.

We re-ran the experiments with the modified JACK and X-JACK and recorded the CPU usage for each run in each system. The difference in times taken to run the same experiments in these two modified systems, indicates the overhead of the additional reasoning in X-JACK. The results are shown in Fig. 17.

We can see from Fig. 17, that there was a slight difference between the CPU usage of X-JACK and JACK. Statistical analysis show that this difference although small was significant. However, on average the difference over the 50 random runs is $0.18 \mathrm{~s}$, which is a small expense for X-JACK successfully achieving twice as many goals on average than JACK (refer Fig. 13).

\subsection{Facilitating Positive Interactions}

The environmental variables that were controlled in these experiments were the amount of potential plan merges, the number of goals pursued in parallel, the depth

\footnotetext{
${ }^{15}$ The GMS was modified to allow all plans to be executed when chosen.
} 
of the plans/sub-goals that get merged, and the duration of the plans of the goals. These variables were chosen for the following reasons:

- The amount of potential merges. The greater the amount of potential merges, the more stressful it would be on the algorithms and data structures. On the other hand, this provides opportunity for greater benefit as well.

- The number of parallel goals. The more goals that execute steps in parallel to each other, the greater the opportunity for merging common steps, if any.

- The depth of the plans/sub-goals that are merged. If a plan or sub-goal that contains a lot of other sub-goals get merged (i.e. replaced by a plan of another goal), the algorithms will realise a greater benefit. For example, in Fig. 7, in the case of the goal of depth 5, there is greater benefit if plan $P 4$ gets merged rather than plan $P 1$.

- The duration of the plans of the goals. Whilst this will not affect the number of plans that get merged, it will affect the run-time costs as when scheduling, a suspended plan would have to wait a longer period of time until the plan it is waiting for is reached.

Our experiments are categorized into the following explorations:

- Run-time costs and benefits of facilitating positive interactions, when there is a high level of possible plan merges;

- Run-time costs and benefits of facilitating positive interactions, when there is a lower, more normal level of possible plan merges;

- The computational cost of the algorithms that manage positive interactions; and

- The effect that varying levels of parallelism have on plan merging.

The experimental setup we used to evaluate these algorithms was identical to the setup we used to evaluate the algorithms for avoiding interference (as described in the previous section), except that instead of the goals interacting negatively, we set up the interactions such that plans between goals could possibly be merged.

To recall the setup, we created 10 different types of goals in terms of the goal/plan structure, and instantiated 20 goals whose type was randomly selected from the 10 goal types. Each experiment was run 50 times, with each run comprising 20 different random combinations of goal instances.

As we did in our earlier experiments, we measured the run-time costs by recording the time taken for all 20 goals to complete. Note that there were no negative interactions present and the goal execution is controlled such that no plans fail. Hence, all 20 goals completed in both JACK as well as X-JACK. The benefit of using X-JACK with the plan merging algorithms was that less plans needed to be executed compared to JACK to achieve the same amount of goals. Therefore, the total number of plans that get executed is also recorded to illustrate the benefits of X-JACK.

\subsection{High Levels of Potential Merges}

Using the setup as above, we simulated a high level of possible plan merges as follows: Of the 10 goal types, we set up five goal types such that each goal type contained two plans that could possibly be merged with plans of two other different goals. The other five goal types contained one plan that could possibly be merged with 

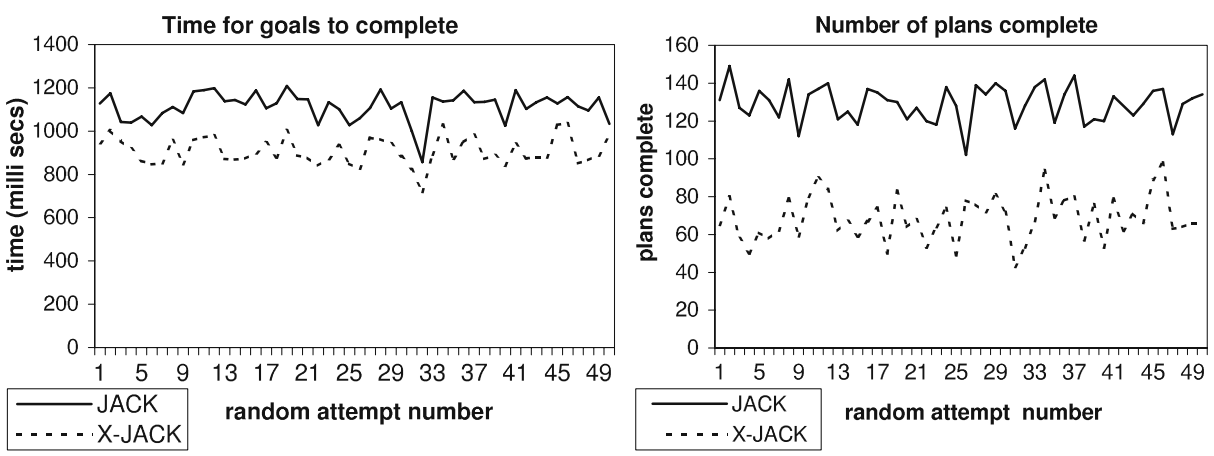

Fig. 18 Positive interactions: results with high levels of possible plan merges

another plan of a different goal. For each goal type, we varied the plans that could potentially be merged, with respect to their position in the goal plan tree. This setup produced an unrealistically high number of possible plan merges, but was useful in testing our algorithms in extreme situations.

The results obtained are shown in Fig. 18, from which it is evident that there is a large benefit in our reasoning methods, in terms of the number of plans executed, when there are a high number of interactions. On average, X-JACK executed $45 \%$ fewer plans than JACK. In terms of the run-time cost, X-JACK performed better as expected, since it executed a lot fewer plans ( $\mathrm{p}$-value $\left.=4 \times 10^{-32}\right)$.

\subsection{Lower/Normal Levels of Possible Plan Merges}

Using the same setup as described above, we simulated a lower, more realistic level of potential merges between the 10 goal types by setting up four of the 10 goal types to not interact with any other goal type, three of the goal types to have two plans that could be merged with plans of different goals, and the other three goal types to have one plan that could be merged with a plan of another goal. We also ensured that the plans that could possibly be merged were at varied positions in their respective goal-plan trees.
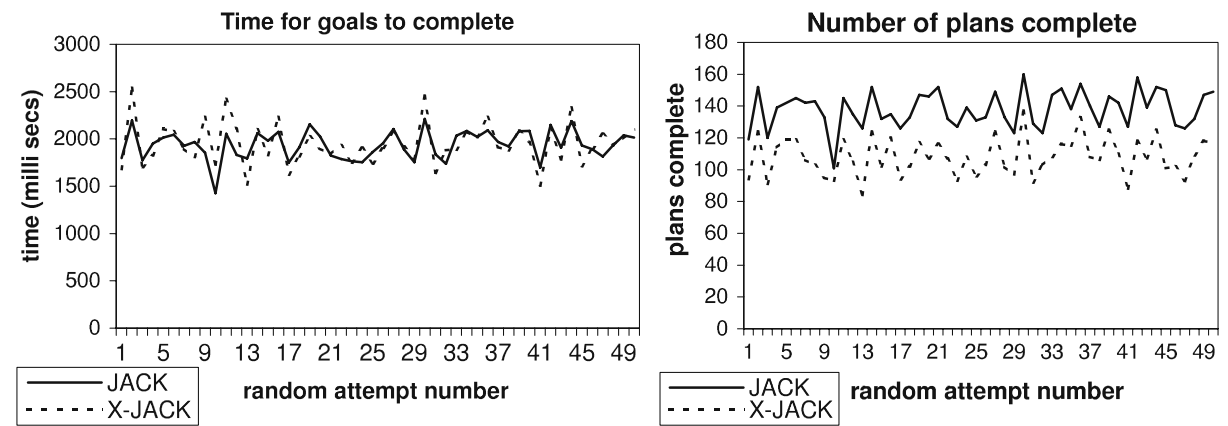

Fig. 19 Positive interactions: results with lower/normal levels of possible plan merges 

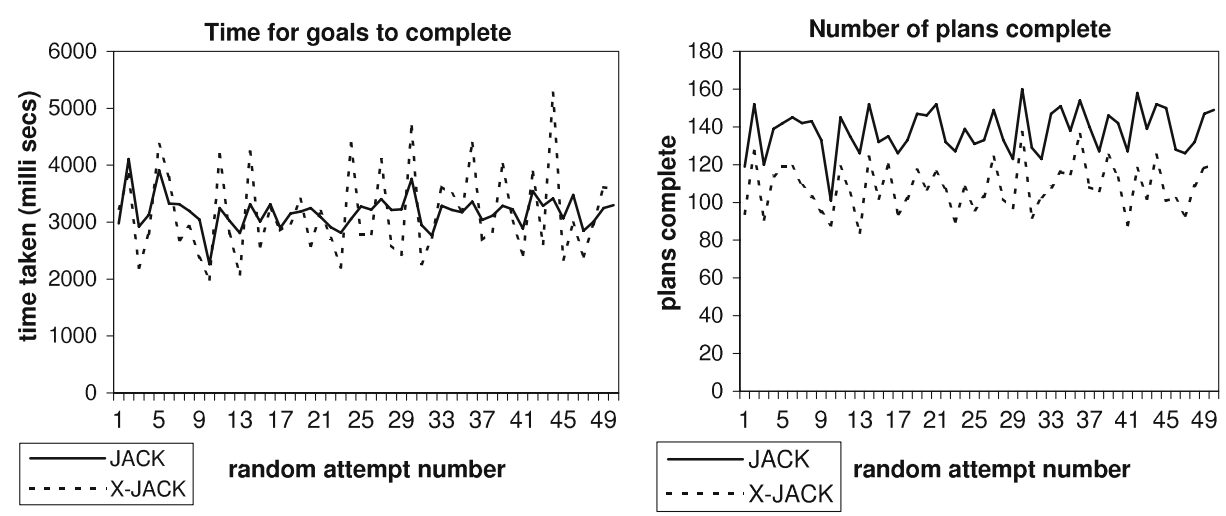

Fig. 20 Positive interactions: results with longer plans

The results are shown in Fig. 19. We see that the benefit was still very evident; with X-JACK on average executing 29 plans less than JACK to successfully satisfy the same amount of goals. The results also showed no significant difference in the run-time costs between the two systems $(\mathrm{p}$-value $=0.62)$. The reason for this is that although X-JACK executed a lesser number of plans, some plans were suspended in $\mathrm{X}$-JACK in order to synchronise for the purpose of merging them with other plans. This incurs a run-time cost.

We then changed the duration of each plan of the goal to double its original duration, and re-ran the experiments. The results shown in Fig. 20. In this case the benefits were almost the same as before. In terms of run-time costs, although the average difference between the two systems was still statistically insignificant ( $\mathrm{p}$-value $=0.71$ ), we notice that in some particular runs, X-JACK took more time than JACK, yet in other situations the converse was true. X-JACK can take more time than JACK on some occasions depending on the amount of time that a plan has to wait, until the plan that it could possibly be merged with is reached. On the other hand, X-JACK may complete faster than JACK on some occasions since there are fewer plans executed by X-JACK compared to JACK.
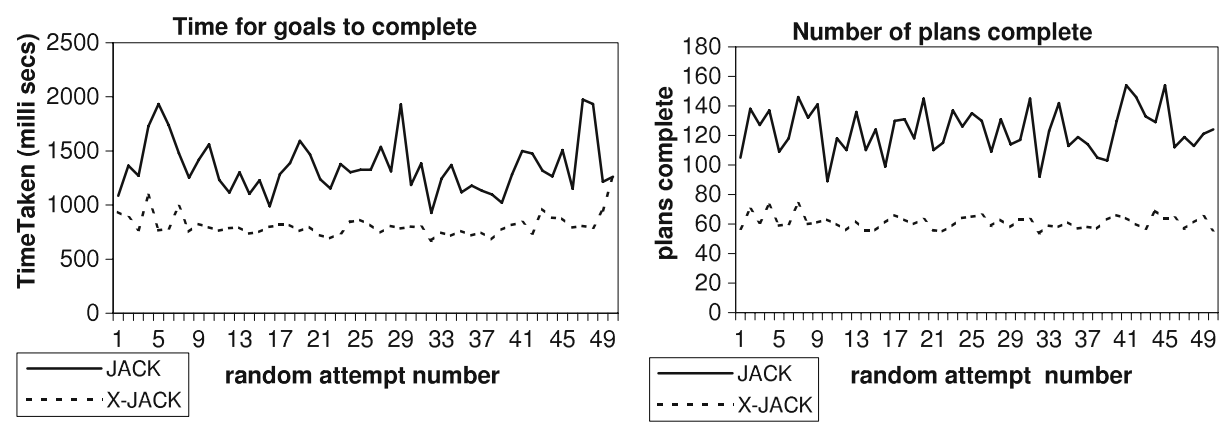

Fig. 21 Positive interactions: situation where sub-goals of greater depth get merged 

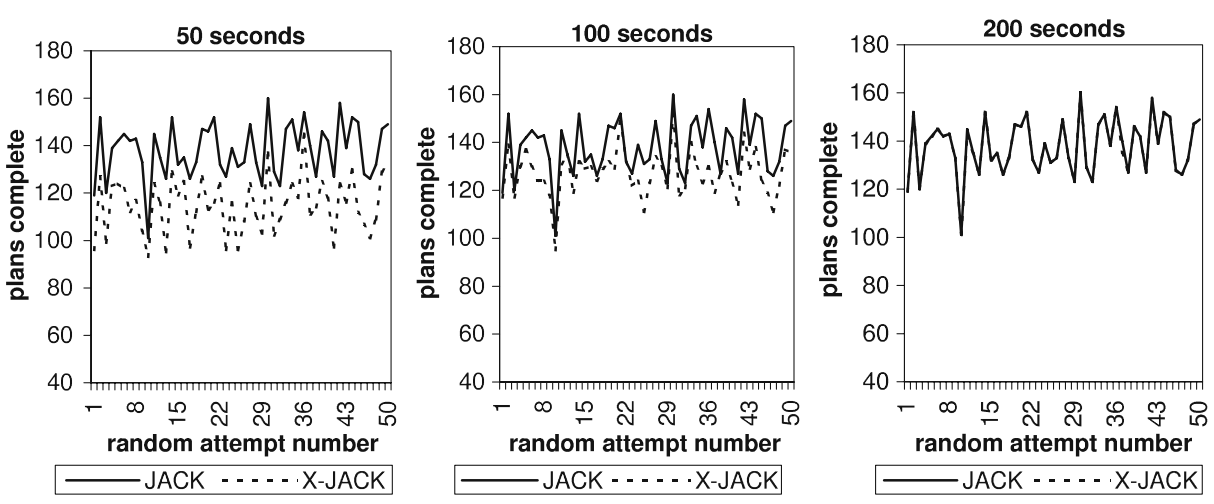

Fig. 22 Positive interactions: effect of adding goals to the system at varying time intervals

In order to observe the impact on the benefit of our algorithms if sub-goals of greater depth (in terms of their (sub)goal-plan tree) get merged, we re-structured the plans such that some of the plans that got merged contained such sub-goals.

The results are shown in Fig. 21. From the figure, it is very clear that there was a much greater benefit in terms of the number of plans that get executed in X-JACK over JACK; on average X-JACK executed half $(50 \%)$ of the amount of plans that JACK did. It is also evident that the run-time costs of X-JACK was much less than JACK, on average X-JACK took $40 \%$ less time than JACK did. As expected, this indicates that our algorithms are most beneficial in situations where potentially mergeable steps are time consuming.

\subsection{Varying Levels of Parallelism}

In order to determine the effect that the amount of goals that occur in parallel has on the amount of plans that get merged, we took the setup for lower/normal levels of potential merges, and added the goals into the system via the GMS at varied time intervals.

Figure 22, shows the results for intervals of length 50, 100 and $200 \mathrm{~ms}$. The graphs show that as the amount of parallelism decreases, the benefit also decreases. The difference in plans executed is still significant in the favour of X-JACK, when the interval is $100 \mathrm{~ms}$ (statistically, $\mathrm{p}$-value $=5 \times 10^{-15}$ ). However, when the interval is $200 \mathrm{~ms}$, although there are a few goal steps that occur in parallel, on average the difference in the number of plans executed is insignificant between the two systems (statistically, $\mathrm{p}$-value $=0.105$ ). Our algorithms are therefore, more beneficial when there is a higher level of parallelism between goals.

\subsection{Computational Cost of Facilitating Positive Interactions}

The previous experiments gave us an indication of the run-time costs but not the computational cost of our algorithms. In order to determine the computational cost, we took the setup where there were high levels of possible plan merges and made the following changes to X-JACK. X-JACK was modified such that the algorithms for identifying plan merges were executed, but the plans were not actually merged 


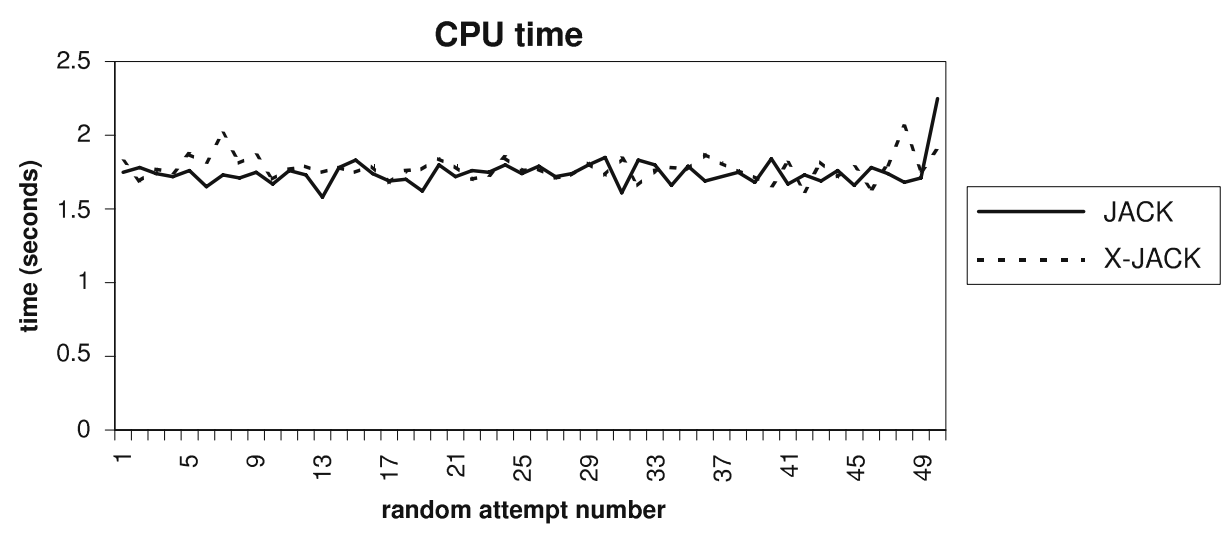

Fig. 23 Positive interactions: computational cost

but allowed to execute normally as they would in JACK. Although the actual plan merging was not done, the process of tracking the effects, delaying plans, and determining which plans to merge was done to reflect the true computation involved.

We re-ran the experiments with JACK and with this modification to X-JACK and recorded the CPU usage for each run in each system. The difference in times obtained was the overhead of the additional reasoning in X-JACK and the results are shown in Fig. 23.

The results show that, the difference in CPU time between X-JACK and JACK over the 50 random runs was statistically insignificant with a p-value of 0.053 . As there was an insignificant overhead and there may be substantial gain, it would seem to make sense to always use these algorithms. Situations where they would not be used in, are where it is important for some goals to be completed as quickly as possible, even if overall time would be saved in delaying the goals.

\section{Conclusion}

The ability to pursue multiple goals is one of the hallmarks of an intelligent agent, and it is important that this is able to be done in a rational way. In previous work we have developed algorithms to do this. In this paper we have implemented these algorithms into the infrastructure of a BDI agent platform, and have evaluated the costs and benefits of this.

We have presented X-JACK, an extension to JACK that incorporates the concept of goals and reasoning algorithms about goal interactions. In order to ascertain the costs and benefits of each of the three different kinds of reasoning we presented, about resource conflicts, interference, and positive interactions between goals, our experiments were conducted individually for each of them. In each case we described the experimental setting including the factors that contribute towards the complexity of the reasoning. We used these settings to explore the costs and benefits of X-JACK over JACK under conditions that were extreme in order to stress-test our algorithms, as well as under conditions that were more realistic. 
Results clearly show that even under extreme circumstances of very high levels of goal interaction, the cost is not excessive for any of the reasoning mechanisms we developed. The benefits however, are very large in such circumstances. In situations where the costs and benefits of our reasoning mechanisms were expected to be at a minimum, while costs were insignificant (statistically at a $0.05 \%$ p-value level), the benefits were still statistically significant.

The results presented here clearly indicate that incorporating the suggested data structures and reasoning algorithms into an agent development toolkit like JACK, allows intelligent agents to be more rational and productive without sacrificing their efficiency.

The techniques we have developed to identify conflicts and synergies can also be used as heuristics to determine which goal or plan to select when there are many applicable in a given situation. This issue and other factors have been discussed elsewhere [22].

The approach described in this work has limitations which we hope to address as future work. Our current work consider only achievement goals, that is goals that achieve a particular state of the world and we assume the goals to be nonrecursive. There are also other types of goals such as maintenance goals, where the agent aims to maintain a particular state of the world [10]. The difficulty with recursive goals is although the interactions in terms of effects can be dealt with using similar techniques to what we have proposed, computing the resource requirements for consumable resources is not possible unless the number of iterations are known. This would require some sort of prediction model which is beyond the scope of this work but indeed an useful future extension. The current representation for resources are simple and don't allow for disjunctions for example. We note that a more sophisticated representation for resources can still be used with our approach which enhances the proposed techniques. For example, Morley et al. [15] have extended some of our early work on resource summary computation to provide a representation and techniques for continually refining the resource requirements of an agent.

The analysis that we have conducted is mostly empirical and would benefit from a theoretical analysis which would also prove the more generalisable nature of the approach. To this end, Shaw and Bordini [21] have adopted the techniques for determining resource conflicts to a Petr-net based reasoning mechanism and performed some practical and theoretical analysis. In their work, the results show clear benefit in using the techniques we have developed even in their Petri-net based implementation. These extensions to our work complements the work presented in this paper.

Acknowledgements We thank Dr. Michael Winikoff for his discussions on the work described in the paper. We would also like to thank Agent Oriented Software Pty. Ltd. for the use of the JACK Intelligent Agents ${ }^{\mathrm{TM}}$ Software.

\section{References}

1. Barber, K.S., Liu, T.H., Goel, A., Martin, C.E.: Conflict representation and classification in a domain independent conflict management framework. In: Proceedings of the Third Annual Conference on Autonomous Agents. ACM Press, Seattle, WA, USA (1999) 
2. Bell, J., Huang, Z.: Dynamic goal hierarchies. In: Doyle, J., Thomason, R.H. (eds.) Working Papers of the AAAI Spring Symposium on Qualitative Preferences in Deliberation and Practical Reasoning, pp. 9-17. American Association for Artificial Intelligence, Menlo Park, California (1997)

3. Booch, G.: Object-oriented Analysis and Design with Application, 2nd edn. Addison-Wesley, Reading (1994)

4. Busetta, P., Bailey, J., Ramamohanarao, K.: A reliable computational model for BDI agents. In: 1st International Workshop on Safe Agents, Held in Conjunction with AAMAS2003 (2003)

5. Busetta, P., Rönnquist, R., Hodgson, A., Lucas, A.: JACK intelligent agents-components for intelligent agents in Java. Technical report, Agent Oriented Software Pty. Ltd, Melbourne, Australia (1998)

6. Clement, B.J., Durfee, E.H.: Identifying and resolving conflicts among agents with hierarchical plans. In: AAAI Workshop on Negotiation: Settling Conflicts and Identifying Opportunities, AAAI Technical Report WS-99-12 (1999)

7. Clement, B.J., Durfee, E.H.: Theory for coordinating concurrent hierarchical planning agents using summary information. In: Proceedings of the National Conference on Artificial Intelligence (AAAI-99), pp. 495-502 (1999)

8. Clement, B.J., Durfee, E.H.: Top-down search for coordinating the hierarchical plans or multiple agents. In: Proceedings of the Third International Conference on Autonomous Agents (Agents'99), pp. 252-259. ACM Press, Seattle, WA, USA (1999)

9. Cox, J.S., Durfee, E.H.: Discovering and exploiting synergy between hierarchical planning agents. In: Proceedings of the Second International Joint Conference on Autonomous Agents and Multiagent Systems (AAMAS 2003), Melbourne, Australia (2003)

10. Duff, S., Harland, J., Thangarajah, J.: On proactivity and maintenance goals. In: 5th International Joint Conference on Autonomous Agents and Multiagent Systems (AAMAS 2006), pp. 858-865. ACM (2006)

11. Hindriks, K., de Boer, F., van der Hoek, W., Meyer, J.-J.C.: Agent programming with declarative goals. In: Intelligent Agents VI-Proceedings of the 7th International Workshop on Agent Theories, Architectures, and Languages (ATAL'2000). Springer, Berlin (2000)

12. Horty, J.F., Pollack, M.E.: Evaluating new options in the context of existing plans. Artif. Intell. 127, 199-220 (2001)

13. Huber, M.J.: JAM: a BDI-theoretic mobile agent architecture. In: Proceedings of the Third International Conference on Autonomous Agents (Agents'99), Seattle, WA, pp. 236-243 (1999)

14. Ingrand, F.F., Georgeff, M.P., Rao, A.S.: An architecture for real-time reasoning and system control. IEEE Expert: Intell. Syst. Appl. 7(6), 34-44 (1992)

15. Morley, D.N., Myers, K.L., Yorke-Smith, N.: Continuous refinement of agent resource estimates. In: 5th International Joint Conference on Autonomous Agents and Multiagent Systems (AAMAS 2006), pp. 858-865. ACM (2006)

16. Pollack, M.E.: Overloading intentions for efficient practical reasoning. Noûs 25(4), 513-536 (1991)

17. Ramamohanarao, T.K., Bailey, J., Busetta, P.: Transaction oriented computational models for multi-agent systems. In: Proceedings of 13th IEEE International Conference on Tools with Artificial Intelligence (ICTAI), Dallas, U.S.A., pp. 11-17 (2001)

18. Rao, A.S., Georgeff, M.P.: Modelling rational agents within a BDI-Architecture. In: Fikes, R., Sandewall, E. (eds.) Proceedings of the Second International Conference on Principles of Knowledge Representation and Reasoning, KR '91', Cambridge, MA, pp. 473-484 (1991)

19. Rao, A.S., Georgeff, M.P.: An abstract architecture for rational agents. In: Proceedings of the Third International Conference on Principles of Knowledge Representation and Reasoning, pp. 439-449. Morgan Kaufmann Publishers, San Mateo, CA (1992)

20. Rumbaugh, J., Blaha, M., Premerlani, W., Eddy, F., Lorensen, W.: Object-Oriented Modeling and Design. Prentice Hall, Upper Saddle River (1991)

21. Shaw, P.H., Farwer, B., Bordini, R.H.: Theoretical and experimental results on the goal-plan tree problem. In: 7th International Joint Conference on Autonomous Agents and Multiagent Systems (AAMAS 2008), IFAAMAS, pp. 1379-1382 (2008)

22. Thangarajah, J., Harland, J., Yorke-Smith, N.: A soft cop model for goal deliberation in a bdi agent. In: Proceeding of the 6th CP Workshop on Constraint Modelling and Reformulation ModRef-07 (2007)

23. Thangarajah, J., Padgham, L., Winikoff, M.: Detecting and avoiding interference between goals in intelligent agents. In: Proceedings of the Eighteenth International Joint Conference on Artificial Intelligence (IJCAI 2003), Acapulco, Mexico (2003) 
24. Thangarajah, J., Padgham, L., Winikoff, M.: Detecting and exploiting positive goal interaction in intelligent agents. In: Proceedings of the Second International Joint Conference on Autonomous Agents and Multiagent Systems (AAMAS 2003), Melbourne, Australia (2003)

25. Thangarajah, J., Winikoff, M., Padgham, L., Fischer, K.: Avoiding resource conflicts in intelligent agents. In: Proceedings of the 15th European Conference on Artifical Intelligence 2002 (ECAI 2002), Lyon, France (2002)

26. van Riemsdijk, B., van der Hoek, W., Meyer, J.-J.C.: Agent programming in dribble: from beliefs to goals using plans. In: Proceedings of the Second International Joint Conference on Autonomous Agents and Multiagent Systems (AAMAS 2003), Melbourne, Australia (2003)

27. Winikoff, M., Padgham, L., Harland, J., Thangarajah, J.: Declarative \& procedural goals in intelligent agent systems. In: Proceedings of the Eighth International Conference on Principles of Knowledge Representation and Reasoning (KR2002), Toulouse, France (2002) 